\title{
Cyclostrophic corrections of AVISO/DUACS surface velocities and its application to mesoscale eddies in the Mediterranean Sea.
}

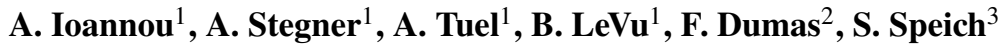 \\ ${ }^{1}$ Laboratoire de Météorologie Dynamique, CNRS, Ecole Polytechnique, Palaiseu, 91128, France \\ ${ }^{2}$ SHOM, Brest, France \\ ${ }^{3}$ Laboratoire de Météorologie Dynamique, CNRS, Ecole Normale Supérieure, 24 Rue Lhomond 75005 Paris, France
}

\section{Key Points:}

- performance of iterative method for recovering cyclogeostrophic balance

- strong cyclostrophic corrections for intense anticyclones in the Mediterranean Sea

- cyclogeostrophic velocity fields in better agreement with in-situ measurements

Corresponding author: Artemis Ioannou, artemis.ioannou@lmd.polytechnique.fr 


\section{Abstract}

Mesoscale eddies, having a characteristic radius equal or larger than the local deformation radius, are generally considered to be geostrophic. Even if this is true for most of them, there are few cases where the ageostrophic velocity components induced by the local curvature of the streamlines are not negligible. In order to account for this ageostrophic part, we investigate the performance of an optimized iterative method which computes the cyclostrophic corrections starting from the geostrophic surface velocity of the AVISO/DUACS. We optimized the convergence of the iterative method using an intermediate cubic interpolation. The performance and the accuracy of the optimized iterative method is first evaluated on idealized eddies for which we can obtain their exact cyclogeostrophic solution. Mesoscale eddies of various shapes, intensities and different ellipticity are investigated. The iterative method is then applied to fifteen years (2000-2015) of AVISO/DUACS geostrophic velocity fields, gridded at $1 / 8^{\circ}$ for the Mediterranean Sea. We found that these ageostrophic corrections are needed for most of the mesoscale anticyclones that have a geostrophic vortex Rossby number larger than $R o>0.1$. Both the Alboran and the Ierapetra eddies are frequently affected by the cyclostrophic corrections that may exceed $50 \mathrm{~cm} \mathrm{~s}^{-1}$. Lastly, the corrected velocity fields are compared with available in-situ observations of velocity measurements (VMADCP) performed within the Ierapetra eddy confirming the benefit of the proposed method.

\section{Introduction}

The increase of the spatial resolution of remote sensing observations has revealed the prevalence of mesoscale eddies throughout the oceans. These coherent structures can survive several months and sometimes several years [Puillat et al., 2002; Ioannou et al., 2017; Laxenaire et al., 2018]. They are able to trap and transport heat, mass, and momentum from their regions of formation to remote areas. However, a correct assessment of eddy properties and how they vary temporally is still a challenge. The existing estimations are derived by analyzing satellite altimetry gridded fields which provide daily global 2D maps of sea surface height and surface geostrophic velocity that are not affected by cloud coverage.

In the last 10 years, eddy detection algorithms have been developed and used to identify automatically ocean mesoscale eddies [Doglioli et al., 2007; Chelton et al., 2007; Chaigneau et al., 2009; Chelton et al., 2011; Nencioli et al., 2010; Mason et al., 2014; 
Le Vu et al., 2018]. These methods locate the eddy center and estimate the eddy size. The eddy intensity is then usually defined as the difference of sea surface height (i.e. hydrostatic pressure gradient) between the eddy center and its periphery [Chaigneau et al. 2009; Chelton et al., 2011; Souza et al., 2011; Mason et al., 2014] or from some dimensionless parameters derived from the eddy surface velocity field: the relative eddy-core vorticity (Doglioli et al. [2007]), the Okubo-Weiss parameter [Isern-Fontanet et al., 2006] or the vortex Rossby number [Mkhinini et al., 2014; Le Vu et al., 2018; Laxenaire et al., 2018]. The main advantage in using the latter is that it is easily comparable with direct in-situ measurements such as VMADCP, LADCP [Ioannou et al., 2017], high frequency radar (HFR) current measurements [Chavanne et al., 2010] or trajectories inferred from surface drifters [Sutyrin et al., 2009; Mkhinini et al., 2014; Ioannou et al., 2017]. However, the derivation of ocean surface velocity from remote sensing altimetry is based on the strong assumption that oceanic currents and, in particular, mesoscale eddies satisfy the geostrophic balance. This approximation is inaccurate for submesoscale structures whose ageostrophy is large [Chang et al., 2013], but it could also induce significant bias for mesoscale eddies.

The dynamical characteristics of small-scale surface eddies $(5-20 \mathrm{~km})$ that were not accessible before with traditional oceanographic campaigns, can now be obtained from high frequency radar (HFR) current measurements [Paduan and Washburn, 2013; Schaeffer et al., 2017] or from an intensive scanning of a small oceanic area with shipboard ADCP [Hasegawa et al., 2004; Chang et al., 2013]. These recent observations of submesoscale eddies, having a radius smaller than the first baroclinic deformation radius, have shown that their relative vorticity $\zeta_{0} / f$, where $\zeta_{0}$ is the surface vorticity measured in the eddy core and $f$ the Coriolis parameter, could exceed unity and could reach values up to $\left|\zeta_{0} / f\right|=5-10$ [Chang et al., 2013]. Such strongly ageostrophic structures which evolve rapidly cannot be detected by the current spatio-temporal resolution of altimetry products and are therefore, out of the scope of this paper.

On the other hand, mesoscale eddies, having a characteristic radius equal or larger than the local deformation radius, are generally considered to be geostrophic. Even if this is true for most of them, there are nevertheless few cases where the ageostrophic velocity components induced by the local curvature of the streamlines are not negligible [Penven et al., 2014; Douglass and Richman, 2015; Ioannou et al., 2017]. To make the distinction with the ageostrophic velocities induced by the surface wind-stress, we use here and in 
what follows, the term cyclostrophic velocity correction for these ageostrophic velocity components which take into account the centrifugal acceleration. The pioneering work of Uchida et al. [1998], has shown that adding small ageostrophic velocity components, induced by the curvature of the Kuroshio, improves the comparison of surface velocities calculated from satellite altimetry (TOPEX/POSEIDON at that time) with the drifting buoys velocities. More recent studies have shown that cyclostrophic corrections should be applied to the geostrophic velocity, derived from altimetry maps, to assess correctly the azimuthal velocity of some intense mesoscale eddies in the Mozambique channel [Penven et al., 2014], or for the intense Gulf stream rings [Douglass and Richman, 2015]. Similarly, in the Mediterranean Sea strong ageostrophic components have been reported for anticyclonic eddies in two specific areas. The Western Alboran Gyre, located between the Strait of Gibraltar and Cape Tres Forcas, constitutes one of the strongest anticyclonic features of the western Mediterranean Sea, with surface currents which exceed $1 \mathrm{~m} / \mathrm{s}$ [Viudez et al., 1996a,b; Gomis et al., 2001; Flexas et al., 2006]. Moreover, in the eastern Mediterranean Sea, the Ierapetra anticyclones (IEs), that recurrently form at the south-east corner of Crete, could also reach finite vorticity values [Matteoda and Glenn, 1996]. The IEs can remain close to the area of their formation but also drift long distances in the Levantine basin ([Hamad et al., 2006; Ioannou et al., 2017]). Strong ageostrophic components were observed along their dynamical evolution. For these two specific areas, in-situ measurements revealed the inadequacy of the geostrophic approximation to describe the eddy dynamics. The standard AVISO/DUACS products, may often underestimate the eddy intensity. However, in the Mediterranean Sea, ageostrophic corrections may not be limited to these two eddies.

Two approaches were used to compute the cyclostrophic velocity corrections on AVISO/DUACS products so far. The first one is to solve the quadratic cyclogeostrophic equation (i.e. Eqn. (7) in subsection 4.1) for circular eddies which were detected in the geostrophic velocity field. It was applied by Ioannou et al. [2017] for a few quasi-circular configurations of the Ierapetra anticyclone and by Douglass and Richman [2015] who assumed a Gaussian shape for all the quasi-circular eddies of the Atlantic ocean. This method is quite simple but it requires to know precisely the velocity profile of the geostrophic eddy and it is valid only for circular eddies. The second one, is based on an iterative method which adds at each step small corrections to the surface velocity field in order to account for the centrifugal acceleration induced by the local curvature [Arnason et al., 
1962; Penven et al., 2014]. The main advantage of this global approach is that it provides a cyclostrophic correction for all eddies regardless their initial shapes. The main drawback is that the iteration may not converge to the exact cyclogeostrophic balance and so careful accuracy tests should be done.

In the present study, we optimized the convergence of the iterative method using an intermediate cubic interpolation. Besides, we tested thoroughly the accuracy of the method on idealized eddies for which we can obtain a direct solution of the cyclogeostrophic balance. We explore a wide distribution of sizes and intensities but also various shapes that correspond to the statistical distribution of mesoscale eddies in the Mediterranean Sea. Then, we applied this cyclostrophic correction to fifteen years (2000-2015) of daily geostrophic velocity fields provided by AVISO/DUACS for the Mediterranean Sea at the high grid resolution of $1 / 8^{\circ}$. We found that it may significantly impact the estimated intensities of mesoscale anticyclones, especially the Alboran and the Ierapetra eddies but not only. Finally, the corrected surface velocity fields were compared with direct in-situ measurements performed within the Ierapetra anticyclone during the PROTEVS-PERLE campaign of October-November 2018.

\section{Data}

\subsection{AVISO data set}

We used in the present study the geostrophic velocity fields, for the years 2000-2015, produced by SSALTO/ Data Unification and Altimeter Combination System (DUACS) and distributed by AVISO and derived from the absolute dynamical topography (ADT). Unlike the seal level anomaly (SLA), which represents the variable part of sea surface height, the ADT is the sum of this variable part and the constant part averaged over a 20 -year reference period. The "all sat merged" series distributed regional product for the Mediterranean Sea combines, up-to-date datasets with up to four satellites at a given time, using all the missions available at a given time [TOPEX/Poseidon, ERS-1 and ERS-2, Jason-1 and Jason-2, the Ka-band Altimeter (AltiKa) on the Satellite with the Argos Data Collection System (Argos) and AltiKa (SARAL), Cryosat-2 and Envisat missions]. This merged satellite product, for the Mediterranean Sea, is projected on a $1 / 8^{\circ}$ Mercator grid, with a time interval of $24 \mathrm{~h}$. 
The spatial resolution of this regional dataset is 2 times higher than the global altimetric products at $1 / 4^{\circ}$. Nevertheless, it remains a coarse-resolution product, because the horizontal resolution of the $1 / 8^{\circ}$ gridded velocity fields $(d X \simeq 12 \mathrm{~km}$ ) cannot fully resolve the internal deformation radius that is around $R d \approx 8-12 \mathrm{~km}$ in the Mediterranean Sea [Robinson et al., 2001; Escudier et al., 2016]. Moreover, the recent analysis of [Amores et al., 2018], which compares the eddies detected on a high-resolution numerical simulation $\left(1 / 60^{\circ}\right)$ with those detected on a synthetic AVISO field $\left(1 / 8^{\circ}\right)$, showed that only eddies, with a characteristic eddy radius smaller than $R_{\max } \leqslant 25 \mathrm{~km}$ (i.e. $R_{\text {end }} \leq 35 \mathrm{~km}$ ) couldn't be correctly detected with the regional AVISO/DUACS dataset. It will be therefore useless to apply any cyclostrophic correction on inaccurate submesoscale structures that may appear on the AVISO field.

\subsection{Shipboard ADCP measurements during the PROTEVS-PERLE campaign}

The PROTEVS-PERLE campaign was held in October-November 2018 in the eastern Mediterranean Sea. Among the various measurements (CTD, LADCP, SEASOR etc.) performed during the PERLE experiment we focus here on the vertical current profiles that were acquired with Ocean Surveyors $150 \mathrm{kHz}$ and $38 \mathrm{kHz}$ (Teledyne RDI) when the Ierapetra eddy was crossed between the 28th of October until the 2nd of November. These systems are two Vessel-Mounted Acoustic Doppler Current Profilers (ADCP) on the R/V L'Atalante. In order to obtain vertical profiles of current speed and direction in the upper layer, we use the $150 \mathrm{kHz}$ Ocean Surveyors that provide velocity measurements every $8 m$ with maximum depth of about $220 \mathrm{~m}$. The first bin sampled is located $26 \mathrm{~m}$ beneath the surface in order to avoid any reflections and interactions with the vessel. The range covered by the OS150 instrument varied between $150 \mathrm{~m}$ and $220 \mathrm{~m}$ over the diurnal cycle. Despite it's short range it provides permanently an assessment of the horizontal components of the current between $26 \mathrm{~m}$ and $100 \mathrm{~m}$. The velocities obtained are averaged over $2 \mathrm{~min}$. The ensemble and the bin size provide a precision of the horizontal velocity that was assessed to be below $8 \mathrm{~cm} \mathrm{~s}^{-1}$. Compared to the velocities observed in the vicinity of the Ierapetra periphery, this corresponds to an error of a bit less than $10 \%$. 


\section{Methods}

\subsection{AMEDA eddy detection algorithm}

In order to quantify the eddy size and their intensity, we apply the Angular Momentum Eddy Detection and tracking Algorithm (AMEDA) which is based on physical parameters and the geometrical properties of the velocity field [Le Vu et al., 2018]. The eddy centers are first identified and correspond to an extremum of the local normalized angular momentum. The streamlines surrounding this center are then computed (Figure 1(b)). The mean radius $\langle R\rangle$ and the mean velocity $\langle V\rangle$ are evaluated for each closed streamline. This mean radius $\langle R\rangle$ is defined as the equivalent radius of a circular disc with the same area A as the one delimited by the closed streamline (Eqn. (1)), while the mean velocity amplitude $\langle V\rangle$ is derived from the circulation along the closed streamline $C$, where $L_{p}$ is the streamline perimeter (Eqn. (2)).

$$
\begin{gathered}
\langle R\rangle=\sqrt{A / \pi} \\
\langle V\rangle=\frac{1}{L_{p}} \oint_{C} V d l
\end{gathered}
$$

We plot in Figure 1(c) the pair of the mean eddy velocity $\langle V\rangle$ and the mean radius $\langle R\rangle$ for each closed streamline of the mesoscale anticyclone located at the east of Sardinia the 2nd of November 2004. We can see on this example that the mean velocity increases when the radius increases until a maximum velocity $V_{\max }$ is reached. The corresponding radius is named $R_{\text {max }}$, also called the speed radius [Chelton et al., 2011; Le Vu et al., 2018; Laxenaire et al., 2018]. The characteristic contour of the detected eddy (blue contours in Figure 1) is associated with the closed streamline of maximal speed. After this maxima, the azimuthal speed of the eddy decreases until the last closed streamline is reached. The latter is plotted with a black dashed line in Figure 1.

From the characteristic eddy velocity $V_{\max }$ and the corresponding radius $R_{\max }$, we compute the vortex Rossby number to quantify the eddy intensity:

$$
R o=\left|\frac{V_{\max }}{f R_{\max }}\right|
$$

where $f$ is the Coriolis parameter. The eddy shape is characterized by two geometrical parameters. The first one is the ellipticity $\varepsilon$ of the closest ellipse that fits the characteristic contour. The second one is the steepness parameter $\alpha$ which is used to fit the mean velocity profile $\langle V\rangle=F(\langle R\rangle)$ of quasi-circular eddies $(\varepsilon<0.2)$. These mean velocity profiles 
are fitted with the generic function:

$$
V_{\theta}(r)=\frac{V_{\max }}{R_{\max }} r e^{\left(1-\left(r / R_{\max }\right)^{\alpha}\right) / \alpha}
$$

Such generic profiles were used by Carton et al. [1989]; Stegner and Dritschel [2000]; Lazar et al. [2013] to study the stability of various isolated eddies. Moreover, Ioannou et al. [2017] found that such generic velocity profile Eqn. (4) provides a high correlation fit for the 22 year analysis of the Ierapetra anticyclones. Note that when $\alpha=2$ the eddy has a Gaussian velocity profile.

We apply the AMEDA algorithm to fifteen years (2000-2015) of surface velocity fields provided by AVISO/DUACS for the Mediterranean Sea. These velocity fields are derived from the absolute dynamical topography (ADT) according to the geostrophic balance. Hence, all the following results are valid for geostrophic structures. The global statistics of the dynamical and geometrical properties of these geostrophic mesoscale eddies, detected by the AMEDA algorithm and having a characteristic radius larger than $18 \mathrm{~km}$, are plotted separately for cyclones and anticyclones in Figure 2. The total number of detected cyclones ( 295000) is slightly larger than the detected anticyclones ( 220000). However, if we consider intense eddies, the proportion is strongly reversed and we get 16600 anticyclones and 5000 cyclones having a geostrophic Rossby number larger than 0.1. For larger values, for instance $R o \geq 0.15$, there is a large predominance of anticyclones as shown in Figure 2(a). A significant cyclone anticyclone asymmetry is also visible on the eddy shape. The mesoscale cyclones tend to be more elliptical than the mesoscale anticyclones. There is a clear predominance of cyclonic structures when the ellipticity $\varepsilon$ exceeds 0.3 (Figure 2(b)). However, as far as quasi-circular eddies are concerned, there is no clear asymmetry for the azimuthal velocity profiles. Both cyclones and anticyclones exhibit a similar distribution of the steepness parameter $\alpha$ which varies between $\alpha=1.2$ and $\alpha=2.7$ while the highest probability is close to the Gaussian shape $(\alpha=2)$. Hence, this statistical analysis of the AVISO/DUACS data set, suggests that there is no universal velocity profile for mesoscale eddies in the Mediterranean Sea. The geostrophic Rossby number could be quite large exceeding 0.2 while a quite large number of eddies deviate from the circular symmetry with a mean ellipticity which exceeds $\varepsilon>0.3$. 


\subsection{Iterative method to compute the cyclogeostrophic velocities}

We consider in what follows large oceanic eddies that evolve and propagate slowly over time. For such mesoscale oceanic eddies, the flow acceleration is negligible in comparison with the centrifugal acceleration induced by the streamlines curvature and therefore the surface velocity field $\mathbf{U}$ should satisfy the cyclogeostrophic balance:

$$
\mathbf{U} . \nabla \mathbf{U}+f \mathbf{k} \times \mathbf{U}=-g \nabla \eta=f \mathbf{k} \times \mathbf{U}_{\mathbf{g}}
$$

where $\mathbf{U}_{\mathrm{g}}$ is the geostrophic velocity which is directly proportional to the gradient of the sea surface deviation $\nabla \eta$.

For the case of a steady circular eddy, this balance relation is strictly identical to the Bolin-Charney balance on a $f$-plane [Charney, 1955]. Higher order balanced equations were proposed for synoptic-scale weather systems which evolve rapidly over a few days in order to account for the divergent components of the flows [Iversen and Nordeng, 1982, 1984; McIntyre, 2015]. However, most of the mesoscale oceanic eddies are, at the first order of approximation, non-divergent and they evolve slowly if we neglect rapid merging and splitting events.

For non-circular eddies there is no analytical solution for $\mathbf{U}$ when $\mathbf{U}_{\mathrm{g}}$ is known. Besides, this non-linear balance may have no solution at all, for instance when the geostrophic Rossby number of a circular anticyclone exceeds the critical value $R o=V_{g} /(f R)>$ $R o_{c}=0.25$ [Knox and Ohmann, 2006; Penven et al., 2014]. However, according to the Figure 2(a) such intense anticyclones are extremely rare (less than $0.01 \%$ ) in the Mediterranean Sea and we therefore expect that the wide majority of mesoscale eddies detected on the AVISO/DUACS database satisfy the cyclogeostrophic balance Eqn. (5).

In order to calculate the ageostrophic velocity components of intense eddies having various shapes and velocity profiles we use an iterative method that was first proposed in atmospheric science [Arnason et al., 1962] and used for intense oceanic eddies in Penven et al. [2014] to approximate the cyclogeostrophic balance Eqn. (5). This iterative scheme is given by:

$$
\mathbf{U}^{\mathbf{n}+\mathbf{1}}=\mathbf{U}_{\mathbf{g}}+\frac{1}{f} \mathbf{k} \times\left(\mathbf{U}^{\mathbf{n}} \cdot \nabla \mathbf{U}^{\mathbf{n}}\right)
$$


where $\mathbf{U}^{\mathbf{0}}=\mathbf{U}_{\mathrm{g}}$. We first project, with a cubic interpolation, the initial geostrophic velocity field gridded at $1 / 8^{\circ}$ on a finer grid at $1 / 24^{\circ}$ in order to improve the computation of the velocity derivatives in Eqn. (6).

There is no proof of convergence for this iterative scheme and for intense eddies it may even diverge after few iterations [Arnason et al., 1962; Penven et al., 2014]. An example of the divergence of the velocity profile, for an initial geostrophic anticyclone with $R o=0.23$, is given in Figure 3(a). Hence, to prevent such local divergence, we performed, as Penven et al. [2014], a constraint iteration which stops the iteration at a grid point when the local residual $\left\|\mathbf{U}^{n+1}-\mathbf{U}^{n}\right\|$ starts to increase. The local norm \|\| is computed here on nine grid points: the central one and the eight closest neighbors. For the example shown in Figure 4, the iteration will stop in the core of the anticyclone after two steps. The Rossby number of the final cyclogeostrophic anticyclone (red curve) will reach $R o=0.48$ which is twice its initial value (Figure 3 ).

\section{Cyclogeostrophic balance of steady and isolated eddies}

In order to test the accuracy of the iterative method (Eqn. (6)) and to develop some algorithmic optimizations, it was needed to compare the results with several test cases. We first consider circular eddies for which we can get simple analytical solutions for both the geostrophic and the cyclogeostrophic balance (Eqn. (5)). Then, assuming a slow evolution of the velocity field, we also consider steady elliptical eddies for the test cases.

\subsection{Impact of the cyclogeostrophic corrections on circular eddies}

Circular eddies are steady solutions of the cyclogeostrophic equation (Eqn. (5)) which simplifies for any azimuthal velocity profile $V_{\theta}(r)$ to the gradient-wind equation:

$$
\frac{V_{\theta}^{2}}{f r}+V_{\theta}=V_{g}=\frac{g}{f} \frac{\partial \eta}{\partial r}
$$

where $V_{g}(r)$ is the geostrophic velocity profile associated to the free surface deviation $\eta$. Cyclonic eddies correspond to $V_{\theta}>0$ while for anticyclonic eddies $V_{\theta}<0$. To study various velocity profiles, we use the generic function (Eqn. (4)) for the azimuthal velocity. The relation between the geostrophic velocity and the cyclogeostrophic velocity will then depend both on the dimensionless Rossby number $R o$ and the steepness parameter $\alpha$. For very small Rossby number, the eddy satisfies the geostrophic balance and therefore 
$V_{\theta} \simeq V_{g}$. However, when the Rossby number starts to increase, the centrifugal acceleration should be taken into account and due to the non-linear term of Eqn. (7) it induces an asymmetry between cyclonic and anticyclonic eddies. Hence, if we compare geostrophic velocities of opposite sign but of the same intensity (i.e. same amplitude of the free surface deviation) the cyclogeostrophic velocity could differ significantly even if the Rossby number is moderate. We should make here the distinction between the geostrophic vortex Rossby number $R o_{g}=\max \left(\left|V_{g}\right|\right) / f R_{\operatorname{maxg}}$ computed from the maximum value of geostrophic velocity and the real Rossby number Ro associated to the complete velocity of the gradient-wind equation (Eqn. (7)). We illustrate in Figure 5 this asymmetry in the cyclogeostrophic correction for some examples of isolated mesoscale eddies the characteristics of which could be observed in the Mediterranean Sea. The comparison is made between eddies of distinct shape (i.e. steepness parameter $1 \leq \alpha \leq 3$ in agreement with Figure 2(c) but with the same size $R_{\max }=30 \mathrm{~km}$ and the same geostrophic amplitude $\max \left(\left|V_{g}\right|\right)=42 \mathrm{~cm} \mathrm{~s}^{-1}$. For these cases the geostrophic Rossby number is moderate $R o_{g}=0.14$ but nevertheless the cyclogeostrophic velocity profiles differ significantly from the geostrophic solution. The amplitudes of anticyclonic (cyclonic) eddies are amplified (attenuated). The maximum velocity of the anticyclones increases up to $V_{\max }=-52 \mathrm{~cm} \mathrm{~s}^{-1}$ while, for cyclones, it decreases slightly down to $V_{\max }=36 \mathrm{~cm} \mathrm{~s}^{-1}$. Moreover, depending on their specific shape, the characteristic radii $R_{\max }$ of the anticyclones (cyclones) are reduced (increased) in comparison with their geostrophic signature.

To investigate a wider range of parameters and quantify more precisely the deviation between the cyclogeostrophic and the geostrophic velocity profiles we plot, for three distinct profiles, the percentage of the relative error on the vortex Rossby number:

$$
\Sigma_{R o}=\frac{R o-R o_{g}}{R o_{g}}
$$

and on the characteristic eddy radius:

$$
\Sigma_{R}=\frac{R_{\max }-R_{\operatorname{maxg}}}{R_{\operatorname{maxg}}}
$$

as a function of the geostrophic Rossby number $R o_{g}$ which is the only dynamical parameter that can be initially deduced from the altimetry data-sets. The cyclostrophic corrections are more pronounced for anticyclonic eddies than for cyclonic ones (Figure 6). The standard geostrophic velocity provided by the AVISO/DUACS products underesti- 
mate the intensity of mesoscale circular anticyclones especially when their geostrophic Rossby number exceeds 0.1 . Besides, this analysis shows that the cyclostrophic correction is indeed profile dependent. The percentage of the cyclostrophic correction depends both on the vortex intensity (i.e. $R o_{g}$ ) and the steepness parameter $\alpha$ of the velocity profile. Hence, the vortex intensity, is not the single parameter that controls the deviation from the geostrophic approximation.

\subsection{Accuracy of the iterative method on circular eddies}

The analytical solutions, obtained in the previous section for circular eddies, can then be used to test the accuracy of the iterative method Eqn. (6). We first project the geostrophic velocity components of the circular vortex on a regular $1 / 8^{\circ}$ grid which is identical to the standard AVISO/DUACS gridded products. In a second step, this geostrophic velocity field is interpolated on a higher resolution grid at $1 / 24^{\circ}$ to improve the computation of the velocity gradients in the non linear terms of the Eqn. (6). The iterative scheme is then applied to this new velocity field and the corrected circular velocity profile is then estimated at each step of the iteration. In order to prevent local divergence, the iteration process is stopped when the local residual $\left\|\mathbf{U}^{n+1}-\mathbf{U}^{n}\right\|$ starts to increase. As shown in Figure 7 this iterative scheme may, or may not, converge to the exact cyclogeostrophic solution but due to the constrain on the decay of local residual it will not diverge. The iteration scheme applied on two Gaussian anticyclones $(\alpha=2)$ with the same radius $\left(R_{\text {maxg }}=30 \mathrm{~km}\right)$ but different intensities is depicted in Figure 7 . When the initial geostrophic Rossby number is moderate $\left(R o_{g}=0.16\right)$ the scheme converges rapidly, after 4 iterations, to the cyclogeostrophic solution (Figure 7(a)). The latter has a smaller radius $\left(R_{\max }=26 \mathrm{~km}\right)$ and a significantly higher Rossby number $(R o=0.24)$ than the initial geostrophic velocity profile. However, when the anticyclone intensity $\left(R o_{g}=0.2\right)$ gets closer to the critical value $R o_{c}=0.25$, the iteration scheme does not succeed to reach the cyclogeostrophic solution and a residual error of $17 \%$ on the Rossby number does persist after 5 iterations (Figure 7(b, c)). Cyclonic gaussian eddies were also tested and we did not find any convergence issue even for large Rossby number up to $R o_{g}=0.2$, which is the largest value found for Mediterranean cyclones. For these intense cyclonic eddies the residual errors of the iteration scheme were below $15 \%$. Other iteration scheme using a relaxation parameter were tested on these few test cases. The convergence is slower with 
this under-relaxation scheme but unfortunately it does not provide a better accuracy (Appendix A: )

We also quantify how the initial interpolation on a finer grid, from $1 / 8^{\circ}$ to $1 / 24^{\circ}$, impacts the iteration scheme. The accuracy of the convergence is tested for three cases: no interpolation (open circle), in other words we stay on the initial AVISO/DUACS grid, a linear interpolation at $1 / 24^{\circ}$ and a cubic interpolation at $1 / 24^{\circ}$ (Figure 8 ). We have found that the cubic interpolation improves significantly the accuracy of the iterative scheme, both for the vortex intensity and its size. Higher order interpolation (quintic) and also higher resolution $\left(1 / 48^{\circ}\right)$ were tested with no significant improvements on the iteration scheme.

The accuracy of this optimized iterative scheme was tested for the wide range of parameters $\left(R o_{g}, R_{\max g}, \alpha\right)$ that were found for mesoscale anticyclonic eddies (Figure 2). The percentage of the relative error on the vortex Rossby number between the geostrophic and the cyclogeostrophic anticyclones were plotted at the initial stage (Figure $9(\mathrm{a}, \mathrm{c})$ ) and at the final stage of the iteration process (Figure $9(\mathrm{~b}, \mathrm{~d})$ ). We arbitrary fix the separation between weak (in green) and strong (in red) errors at 30\%. The relative errors are almost negligible $(<15 \%)$ when the vortex Rossby number is below 0.08 . However, when $R o_{g}$ exceeds $0.12-0.15$ the deviations between the cyclogeostrophic and the geostrophic solution becomes strong (i.e. $>30 \%$ ) and the use of the standard AVISO/DUACS geostrophic velocity field will lead to a systematic underestimation of the intensity of circular anticyclones. This deviation tends to decrease when the steepness parameter $\alpha$ increases (Figure 9(b)). For almost all the anticyclones we studied, the iterative scheme reduces this initial deviation and the final result is much closer to the cyclogeostrophic solution than the initial one. Hence, we've shown here that the iterative scheme leads to a correct estimation of the ageostrophic terms, induced by the streamline curvature, for idealized circular eddies.

The $\left(R_{\max }, \alpha\right)$ parameter space was not thoroughly investigated for cyclonic eddies because, their maximal amplitudes are generally weaker than the anticyclones in the AVISO/DUACS product (Figure 2). Besides, for the same geostrophic Rossby number the cyclostrophic correction is generally much weaker for cyclonic eddies than for anticyclonic ones (Figure 6(a)). 


\subsection{Accuracy of the iterative method on elliptical eddies}

We have seen, in the previous section, that the iterative scheme provides a correct estimation of the full cyclogeostrophic profile for idealized circular anticyclones which are not too close to the divergent limit $R o_{g}=0.25$. However, according to the AVISO DUACS products, most of the detected eddies are elliptical (Figure 2(b)). Hence, the accuracy of the iterative scheme should also be tested on an elliptical eddy configuration. We first generate elliptical velocity fields which are non-divergent (i.e. $\nabla . \mathbf{V}=\mathbf{0}$ ). To do so, we started from a circular Gaussian velocity profile $(\alpha=2)$ and we apply a deformation, that conserves the area inside each streamline:

$$
x^{\prime}=x \sqrt{1-\varepsilon}
$$

$$
y^{\prime}=y / \sqrt{1-\varepsilon}
$$

This deformation will transform a circle of radius $R_{\max }$ into an ellipse of ellipticity (i.e. flattening) $\varepsilon$ having for the semi-major $R / \sqrt{1-\varepsilon}$ and the semi-minor axis $R \sqrt{1-\varepsilon}$. We should then also transform the velocity field according to

$$
\begin{aligned}
& V_{x}^{\prime}\left(x^{\prime}, y^{\prime}\right)=\sqrt{1-\varepsilon} V_{x}(x, y) \\
& V_{y}^{\prime}\left(x^{\prime}, y^{\prime}\right)=V_{y}(x, y) / \sqrt{1-\varepsilon}
\end{aligned}
$$

in order to get a non-divergent velocity field and the conservation of angular momentum for each fluid parcel. Such type of deformation could be induced in the real ocean by the external strain exerted on a circular eddy by its close neighbors.

We compute from the elliptical velocity field the geostrophic velocity components according to cyclogeostrophic balance Eqn. (5). Then we apply the iterative scheme Eqn. (6) (with constrain on the local residual) to these geostrophic velocity components and check how close they are to the initial cyclogeostrophic solution. We compare in Figure 10 the vortex Rossby numbers associated to the initial elliptical vortex (black square), to the corresponding geostrophic vortex (open square) and the results of the iterative scheme after five steps (crosses). We study here the impact of the ellipticity $\varepsilon$ while the vortex Rossby 
number is kept fixed to $R o_{g}=0.18$. This initial value is quite large and the cyclostrophic corrections are therefore significant. Indeed, the vortex Rossby numbers of the cyclogeostrophic eddies are almost the double $(0.28<R o<0.3)$ of the initial geostrophic ones. The differences between the cyclogeostrophic solutions and the results of the iterative scheme remain small (less than 15\%) and weakly impacted by a moderate ellipticity. We can see that the intensity (i.e. the Rossby number) of the elliptical structure obtained with the iterative scheme, after five iterations, is relatively close to the cyclogeostrophic one unless the ellipticity exceeds large values (above $\varepsilon>0.6$ ). The agreement is even better when the intensity of the elliptical structure is weaker (not shown here). Hence, the methodology used in this paper to approximate the cyclostrophic velocity components provides accurate results for both circular and elliptical eddies.

\section{Cyclostrophic corrections of mesoscale eddies in the Mediterranean Sea}

\subsection{Statistical analysis}

We now apply the iterative scheme to fifteen years (2000-2015) of surface geostrophic velocity fields provided by AVISO/DUACS for the Mediterranean Sea. Then we use the AMEDA algorithm to detect and track eddies on the corrected velocity field in order to quantify the impact of cyclogeostrophy on the Mediterranean eddies. The statistical properties of the mesoscale eddies (i.e. $R_{\operatorname{maxg}}>18 \mathrm{~km}$ ) of the initial geostrophic eddy field are compared to the mesoscale eddies detected on the new cyclogeostrophic velocity field. As expected the cyclostrophic correction mainly impacts the mesoscale anticyclones (Figure 11). We should note that ageostrophic submesoscale eddies cannot be detected on the AVISO/DUACS altimetry products and therefore only large mesoscale eddies $\left(R_{\max }>R_{d}\right)$ are considered in this analysis.

The probability distribution functions of the vortex Rossby numbers, for both cyclones and anticyclones, are impacted by the cyclostrophic correction when $R o_{g}>0.1$. However, the impact is much stronger for anticyclonic eddies for which the maximum intensities of the probability distribution function almost double (Figure 11(a)) and reach values up to $R o=0.4$. While for cyclonic structures, the maximum intensities of cyclogeostrophic eddies are slightly attenuated in comparison with the geostrophic ones (Figure $11(\mathrm{c}))$. 
Besides, as we have seen on idealized circular eddies (Figure 7) the cyclostrophic corrections also modify the velocity profile, especially in the core of anticyclones, where it may significantly amplify the core vorticity [Ioannou et al., 2017]. This change on the velocity profiles is also visible on the distribution of the steepness parameter $\alpha$. The probability distribution of mesoscale anticyclones is shifted toward lower value of $\alpha$ (Figure 11(b)) while it remains unchanged for cyclones (Figure 11(d)). Lower values of the steepness parameter correspond to a steeper velocity gradient in the eddy core (i.e. stronger core vorticity) and a lower velocity decay at the eddy periphery.

\subsection{Areas where cyclostrophic corrections are significant}

The statistical analysis provides an overall view of the impact of the cyclostrophic corrections but does not allow to identify the areas in the Mediterranean sea where this correction is the most needed. Therefore, we plot in the Figure 12(a), at each grid point, the maximal amplitude of the cyclostrophic correction $\left\|\mathbf{V}-\mathbf{V}_{g}\right\|$ during the 2000 - 2015 period. We plot here the amplitude of this correction (i.e. the difference between cyclogeostrophic and geostrophic velocities) only if it exceeds $10 \mathrm{~cm} \mathrm{~s}^{-1}$. This graph allows us to immediately identify two "hot spots" where the cyclostrophic correction may exceed $50 \mathrm{~cm} \mathrm{~s}^{-1}$. These two places correspond to the usual locations of the Alboran and the Ierapetra anticyclones.

The Alboran eddy is generated by the recirculation of the incoming jet of Atlantic Water (AW) flowing continuously through the Strait of Gibraltar due to the differential pressure gradient that exists between the Mediterranean Sea and the Atlantic Ocean across the Gibraltar Strait. This intense anticyclone constitutes the strongest dynamical feature of the Western Mediterranean mean circulation, with surface currents of up to $1.5 \mathrm{~m} \mathrm{~s}^{-1}$ [Viudez et al., 1996a,b; Gomis et al., 2001; Flexas et al., 2006]. It is therefore normal to observe a strong ageostrophic component in the velocity or the vorticity field [Viudez, 1997]. The analysis of Gomis et al. [2001] has already showed the existence of large ageostrophic velocities up to $40 \mathrm{~cm} \mathrm{~s}^{-1}$, induced by the cyclostrophic acceleration of this intense mesoscale anticyclone. Our analysis shows that these ageostrophic components of the velocity field can be even stronger (Figure 12(a)).

The Ierapetra Eddy (IE), which is generally formed during the summer months at the south-east corner of Crete, is one of the strongest anticyclones of the Eastern Mediter- 
ranean Sea. This first estimation of the core vorticity of the Ierapetra anticyclones, performed by Matteoda and Glenn [1996], was relatively large in comparison with the local Coriolis parameter $f$. More recently, Mkhinini et al. [2014] and especially Ioannou et al. [2017] performed a thorough study of the IEs intensities based on the AVISO/DUACS surface velocity fields. Assuming a circular eddy shape, Ioannou et al. [2017] computed the cyclogeostrophic velocity profiles of the IEs during their formations or intensification stages and found that the core vorticity $\zeta_{0}$ could sometimes exceed the standard threshold of inertial instability $\zeta_{0}<-f$. Hence, it is not surprising that the cyclogeostrophic corrections, computed by the iterative scheme, are very strong in the formation or intensification area of the IEs.

We quantify in Figure 12(a) the amplitude of the cyclostrophic corrections on the velocity magnitude but we could also consider how these corrections impact the intensity of the detected eddies. We plot in Figure 12(b) the location of all the anticyclones having a Rossby number higher than 0.2 after the cyclostrophic correction. This simple criterion selects intense anticyclones which satisfy the cyclogeostrophic balance with finite core vorticity (i.e. $\zeta / f<-0.6$ for circular Gaussian eddies). About 5000 eddy detections satisfy this criterion during the 15 year period. Since we consider here daily detections, several points could correspond to the same eddy. The large majority of these cyclogeostrophic eddies correspond to the Alboran gyres $(60 \%)$ or the Ierapetra anticyclones $(30 \%)$. However, apart from these two "hot spots" it appears that few other anticyclones may also show strong deviations from the standard geostrophic balance in the Mediterranean Sea. Two other areas are concerned: the Algerian basin and a fraction of the Levantine basin, off the Libyo-Egyptian coast.

The first area concerned in the Algerian basin corresponds to the detachment and the recirculation area of long-lived anticyclones named Algerian Eddies (AE) [Escudier et al., 2016; Pessini et al., 2018; Garreau et al., 2018]. These large mesoscale anticyclones, that are formed by the meanders of the Algerian Current, are generally considered to have small Rossby numbers and satisfy the geostrophic balance.

We show in Figure 13(a) the temporal evolution of the Rossby number Ro and the relative core vorticity for an AE detected in 2005. This anticyclone was studied by [Pessini, 2019] and exhibit a significant intensification when it interacts with the Balearic front six months after its formation. During this event, the geostrophic Rossby number reaches 
large value up to 0.2 which indicates that the cyclogeostrophic balance should be taken into account. Then, when the cyclostrophic correction is applied the vortex Rossby number exceeds 0.3 and the core vorticity could reach intense negative values below $-f$ for several days or weeks.

The second area is located in the Levantine basin $\left(31^{\circ}-34^{\circ} \mathrm{N}, 27^{\circ}-30^{\circ} \mathrm{E}\right)$, and overlays the Herodotus Trough. It has been poorly studied and has very few in-situ observations. Nevertheless, Mkhinini et al. [2014] have shown that the Herodotus Trough is a formation area of long-lived anticyclones. These mesoscale anticyclones, often called MersaMatruh Eddies, have been mentioned in several studies [Horton et al., 1994; Hamad et al., 2006; Amitai et al., 2010; Menna et al., 2012] but never identified as intense eddies. The instabilities of the Libyo Egyptian Current or the local changes of the mean shelf slope could explain the formation of intense meanders or coastal anticyclones in this area.

This analysis confirms that the Alboran and the IE anticyclones are the most intense mesoscale eddies in the Mediterranean Sea. However, the cyclostrophic correction applied to the whole Mediterranean Sea revealed that few other mesoscale eddies that were not identified before as ageostrophic could also exhibit a strong negative core vorticity during their lifetime.

\subsection{Comparison with in-situ measurements}

This study would not be complete without a comparison with in-situ data, to verify that the proposed method effectively corrects the AVISO/DUACS fields so that they are closer to the observations. This requires two conditions that are not easy to obtain during oceanographic surveys. The first one is to find an intense eddy for which the cyclogeostrophic correction will be significant and the second one is to locate accurately the eddy center in order to perform enough velocity measurements within the eddy core. One of the goals of the Atalante cruise during the last PROTEVS/PERLE campaign, held in October-November 2018, was to survey thoroughly the Ierapetra anticyclone in autumn, when its intensity is usually strong. Among the large amount of measurements performed during this campaign, we focus our analysis on two VMADCP transects, which were performed close to the eddy center on October 29th (Figure 14) and on November 1 (Figure 15). First, the geostrophic surface velocities, provided by AVISO/DUACS, were interpolated along the boat trajectory and compared to the VMADCP averaged between $30 \mathrm{~m}$ 
and $70 \mathrm{~m}$. For these two transects (Figure 14(a) and Figure 15(a)), the magnitude of the geostrophic velocity vectors (black arrows) are significantly weaker than the measured ones (blue arrows) while their directions are almost similar. Indeed, the magnitude of the strongest geostrophic velocity component does not exceed $42 \mathrm{~cm} \mathrm{~s}^{-1}$ while the maximum surface velocity measured by the VMADCP reaches $62 \mathrm{~cm} \mathrm{~s}^{-1}$. Thanks to the AMEDA algorithm, the characteristic contours of the geostrophic anticyclone were computed (black contour) and the characteristic speed radius was estimated around $R_{\max } \simeq 34 \mathrm{~km}$. Hence, we can estimate the geostrophic vortex Rossby number $R o_{g} \simeq 0.13$ for this Ierapetra anticyclone. According to our analysis (Figure 9) for such values of the geostrophic Rossby number, the cyclostrophic corrections will be significant. Therefore, in a second step we apply the iterative corrections Eqn. (6) to the surface velocity field and we compare these new velocity fields (in red) to the in-situ measurements (in blue). We observe in the Figure 14(b) and Figure 15(b) better agreements with the observations despite a clear difference in the position of the eddy center. We should mention here that the accuracy of the AVISO/DUACS products is affected by the spatio-temporal distribution of the altimetry tracks and the correlation lengths used in the interpolation scheme [LeTraon et al., 1998] to build the gridded maps from multiple satellites. It is thus, not surprising to find a shift of the order of $10 \mathrm{~km}\left(\sim 1 / 8^{\circ}\right)$ in the positioning of the eddy center. Nevertheless, the maximal amplitude of the cyclogeostrophic velocity field reaches $59 \mathrm{~cm} \mathrm{~s}^{-1}$ which is in better agreement with the observation. The speed radius of the corrected anticyclone is reduced $\left(R_{\max } \simeq 30 \mathrm{~km}\right)$ which leads to a strong increase of the vortex Rossby number up to $R o \simeq 0.2$. Besides, according to the fine comparison of the meridional and latitudinal velocity profiles, plotted in the panels (c, d) of the Figure 14 and Figure 15, we do see that the iterative method improves significantly the velocity gradients in the eddy core. In order to perform relevant comparisons between the VMADCP measurements and the velocities profiles from the geostrophic and cyclogeostrophic fields, any misalignments of the velocity profiles were first minimized. The Root Mean Square Error (RMSE) between the VMADCP measurements and the velocity profiles (geostrophic and cyclogeostrophic) were then estimated. The RMSE of the velocity norm based on the cyclogeostrophic profiles was estimated of the order $9 \mathrm{~cm} / \mathrm{s}$ and $8 \mathrm{~cm} / \mathrm{s}$ for each transect respectively. The cyclogeostrophic RMSE remained $30-40 \%$ lower than the geostrophic one $(13 \mathrm{~cm} / \mathrm{s}$ and $14 \mathrm{~cm} / \mathrm{s})$. 
This example shows that the cyclostrophic corrections of the AVISO/DUACS surface velocities, that we used, are relevant for intense mesoscale anticyclones. Thanks to the optimized iterative method we obtained corrected velocity fields that were much closer to the in-situ observations.

\section{Conclusions}

This study investigates the cyclogeostrophic balance of intense mesoscale eddies in the Mediterranean Sea. To do so, we optimized an iterative scheme, that was initially developed for atmospheric flows [Endlich, 1961; Arnason et al., 1962] and recently used for oceanic eddies in the Mozambique Channel [Penven et al., 2014]. This iterative method computes with the best accuracy the cyclostrophic terms from the geostrophic surface velocity of the AVISO/DUACS products. We have tested the performance of this method on a wide range of idealized mesoscale eddies of different sizes, intensities and shapes that can be detected in the Mediterranean Sea. Since, we can obtain exact cyclogeostrophic solutions for these analytical eddies, we were able to compare the results obtained at the end of the iterations with the exact solutions and therefore validate the accuracy of the whole methodology. The thorough analysis of the various eddy parameters show that the amplitude of the cyclostrophic corrections depend not only on the vortex intensity but also on the vortex shape: the steepness parameter $\alpha$ or the vortex ellipticity $\varepsilon$ for instance. The main advantage of this type of iterative method is that cyclostrophic corrections can be calculated for a very wide range of vortices of different shapes, be they circular or moderately elliptical.

We found that the cyclostrophic correction is needed for most of the mesoscale anticyclones that have a geostrophic vortex Rossby number larger than $\operatorname{Ro}_{g}=\max \left(\left|V_{g}\right|\right) / f R_{\operatorname{maxg}}>$ 0.1. This threshold is below the one chosen by Douglass and Richman [2015]. Indeed, these authors used the value of the mean relative vorticity $\bar{\zeta} / f$ inside the eddy contour to quantify the vortex intensity instead of the vortex Rossby number. For Gaussian eddies, we get the simple relation $R o_{g}=\bar{\zeta} / 2 f$ and therefore the threshold $\bar{\zeta} / f=0.3$ proposed by Douglass and Richman [2015] to classify strong cyclogeostrophic eddies correspond to $R o_{g}=0.15$. The lower value, that we propose, for this correction threshold, is also justified by the intensive survey of the Ierapetra eddy performed during the 2018 PROTEVSPERLE campaign. Even if the initial vortex Rossby number of this mesoscale anticyclone seems week $R o_{g} \simeq 0.13$, below the threshold proposed by Douglass and Richman [2015], 
the corrections that we applied to the AVISO/DUACS geostrophic velocities were significant (54\%) and the corrected velocities were much closer to the VMADCP measurements.

We apply this cyclostrophic correction to fifteen years (2000-2015) of AVISO/DUACS geostrophic velocity fields, gridded at $1 / 8^{\circ}$ for the Mediterranean Sea. We found that velocity errors up to $50 \mathrm{~cm} \mathrm{~s}^{-1}$ could occur for large and intense anticyclones, due to the initial geostrophic approximation. The two most intense anticyclones of the Mediterranean Sea, the Alboran and the Ierapetra eddies, should be corrected but not only. Our analysis suggest that other anticyclones in the Algerian basin or the Levantine basin may also benefit from this ageostrophic correction. The statistical analysis shows that this cyclostrophic correction have a strong impact on the most intense mesoscale anticyclones while it is quite weak for cyclonic eddies. This may seem surprising because in high resolution numerical simulations the most intense and ageostrophic eddies are generally cyclonic [Klein et al., 2008; Roullet and P., 2010; Qiu et al., 2014]. But, we must not forget, that these very intense cyclones correspond to submesoscale eddies, whose radii are less than the local deformation radius, which is around $R_{d}=8-12 \mathrm{~km}$ in the Mediterranean Sea. Since, the effective resolution of altimetric products is coarse, such intense submesoscale cyclones cannot be resolved by the standard AVISO/DUACS regional products gridded at $1 / 8^{\circ}$ [Amores et al., 2018]. Therefore, only large mesoscale cyclones can be detected on altimetry products and they are generally less stable and coherent than their anticyclonic counterpart Stegner and Dritschel [2000]. Several stability analysis have shown that ageostrophic effects, finite Rossby numbers or finite isopycnal deviations, tend to increase the baroclinic instability for cyclones and weaken it for anticyclones [Dewar and Killworth, 1995; Baey and Carton, 2002; Benilov and Flanagan, 2008; Lahaye and Zeitlin, 2015; Mahdinia et al., 2017]. On the other hand, surface intensified anticyclones could remain stable to baroclinic or centrifugal instabilities, even if they reach finite Rossby numbers up to $R o=0.4$ [Lazar et al., 2013].

Such methodology could be easily applied to other sub-basins or marginal seas at mid-latitude in order to improve substantially the estimation of surface velocities. The accuracy of these cyclostrophic corrections depends on the initial resolution of the AVISO/DUACS products and is therefore more relevant on altimetry products gridded at $1 / 8^{\circ}$. These regional products will be more numerous in the years to come, thanks to the growing number of conventional nadir altimeter satellites that will be deployed in the next two years (Jason-C, HY-2C, HY-2D, HY-2E). We could then expect "all sat merged" series at higher 
resolution that will combines up to 5 or 7 altimeters, for several years, with a significant reduction of the inter-track distance. Besides, the operational development of SWOT mission (launched in 2021) will provide wide-swath altimetric measurements of the ocean surface topography leading to an unprecedented increase of the sea surface signature of oceanic mesoscale and submesoscale eddies.

\section{A: Appendix}

We quantify in this section how another iterative scheme that uses an under-relaxation factor $\lambda$ [Iversen and Nordeng, 1982, 1984] may improves on the accuracy of convergence to the cyclogeostrophic solution. In accordance with Figure 7, the accuracy of convergence was tested for two Gaussian anticyclones $(\alpha=2)$ of the same radius $\left(R_{\operatorname{maxg}}=\right.$ $30 \mathrm{~km})$ but different initial geostrophic intensities $\left(R o_{g}=0.16\right.$ and $\left.R o_{g}=0.2\right)$. Based on the iterative method with under-relaxation, only a fraction $\lambda$ of the previous correction is applied at each iteration step (Eqn.(A.1)-Eqn.(A.2)). The iterative scheme with underrelaxation writes as follows:

$$
\mathbf{U}^{\mathbf{n}+\mathbf{1}}=\mathbf{U}_{\mathbf{g}}+\frac{1}{f} \mathbf{k} \times\left(\mathbf{U}^{\mathbf{n}} \cdot \nabla \mathbf{U}^{\mathbf{n}}\right)
$$

$$
\begin{aligned}
\mathbf{U}_{\text {under-relaxation }}^{\mathbf{n}+\mathbf{1}} & =\mathbf{U}^{\mathbf{n}}+\lambda\left(\mathbf{U}^{\mathbf{n}+\mathbf{1}}-\mathbf{U}^{\mathbf{n}}\right) \\
& =(1-\lambda) \mathbf{U}^{\mathbf{n}}+\lambda \mathbf{U}^{\mathbf{n}+\mathbf{1}}
\end{aligned}
$$

\footnotetext{
When $\lambda=1$, there is no under-relaxation and we recover the classical iterative method that was used in this study (full correction at each iteration step). High $\lambda$ parameters provide lower weight to the solution of step $\mathbf{U}^{\mathbf{n}}$. We compare in Figure A.1 the accuracy of the under-relaxation scheme to converge at the corresponding analytical cyclogeostrophic solution for the two anticyclones. Two under-relaxation factors were tested $(\lambda=0.4$ and $\lambda=0.6$ ). The relative error $\Sigma_{R o}$ is computed at each iteration step for the free (Figure A.1(a,d)) and the constrained iterative method (Figure A.1(c,f)). The normalized residual drop $\left\|\mathbf{U}^{n+1}-\mathbf{U}^{n}\right\|$ of the velocity norm illustrates the convergence of the geostrophic
} 
field to the cyclogeostrophic solution at each iteration step (Figure A.1(b,e)). As mentioned in section 4.2, in order to prevent local divergence, the iteration process is constrained when the local residual $\left\|\mathbf{U}^{n+1}-\mathbf{U}^{n}\right\|$ starts to increase. For the eddy example with moderate initial geostrophic Rossby intensity $\left(R o_{g}=0.16\right)$ in Figure A.1(a-c), all iterative schemes converge with high accuracy to the same cyclogeostrophic solution. The relative error $\Sigma_{R o}$ remains below $5 \%$. The under-relaxation delays the iterative method convergence requiring more iterations to reach the same final solution. Yet it does not prevent the local divergence (Figure A.1(b)). The performance of the iterative method is also shown for the eddy example with the strong intensity $\left(R o_{g}=0.2\right)$ in Figure A.1(d-f). Similarly, in this case the convergence is slower but the iterative scheme does not succeed to reach the cyclogeostrophic solution. The residual errors for the constrained iterative method with under-relaxation are estimated $23 \%$ and $29 \%$ for $\lambda=0.6$ and $\lambda=0.4$ respectively. The under-relaxation iterative scheme does not provide for a better accuracy while the residual errors are estimated slightly higher than the standard iteration scheme (17\% when $\lambda=1)$.

\section{Acknowledgments}

This work was funded by the ANR-Astrid Project DYNED-Atlas (ANR-15-ASMA-000301). The gridded altimeter products were produced by SSALTO/DUACS and distributed by the Copernicus Marine Environment Monitoring Service (marine.copernicus.eu). The authors gratefully acknowledge the Délégation Générale de l'Armement which funded the program Protevs II into which the Protevs-Perle campaign was scheduled, the French Naval Hydrologic and Oceanographic Service (SHOM) and the crew of the R/V L'Atalante for their contribution to the PROTEVS-PERLE campaign providing us with the in-situ data. Thanks to the two reviewers for their constructive comments and helpful suggestions on an earlier version of the manuscript. The cyclostrophic data used in this work are stored online (doi:10.5281/zenodo.3452045). The DYNED-Atlas data base for the Mediterranean Sea is available at doi:10.14768/2019130201.2.

\section{References}

Amitai, Y., Y. Lehahn, A. Lazar, and E. Heifetz (2010), "Surface circulation of the eastern Mediterranean Levantine basin: Insights from analyzing 14 years of satellite altimetry data", Journal of Geophysical Research, 115(C10), C10,058, doi:10.1029/2010JC006147. 
Amores, A., G. Jordà, T. Arsouze, and J. Le Sommer (2018), "Up to what extent can we characterize ocean eddies using present-day grid-ded altimetric products?", Journal of Geophysical Research: Oceans, 123, https://doi.org/10.1029/2018JC014140.

Arnason, G., G. J. Haltiner, and M. J. Frawley (1962), “HIGHER-ORDER GEOSTROPHIC WIND APPROXIMATIONS", Monthly Weather Review, 90(5), 175185, doi:10.1175/1520-0493(1962)090<0175:HGWA>2.0.CO;2.

Baey, J., and X. Carton (2002), "Vortex multipoles in two-layer rotating shallow-water flows", J. Fluid Mech, 460, 151-175.

Benilov, E., and J. Flanagan (2008), “The effect of ageostrophy on the stability of vortices in a two-layer ocean", Ocean Modelling, 23, 49-58, doi:10.1016/j.ocemod.2008.03.004.

Carton, X. J., G. R. Flierl, and L. M. Polvani (1989), “The Generation of Tripoles from Unstable Axisymmetric Isolated Vortex structures", Europhysics Letters, 9(4), 339-344, doi:10.1209/0295-5075/9/4/007.

Chaigneau, A., A. Gizolme, and C. Grados (2009), "Mesoscale eddies off peru in altimeter records: Identification algorithms and eddy spatio-temporal patterns", Progress in Oceanography, 79, 106-119, https:// doi.org/10.1016/j.pocean.2008.10.013.

Chang, M.-H., T. Y. Tang, C.-R. Ho, and S.-Y. Chao (2013), "Kuroshio-induced wake in the lee of Green Island off Taiwan", J. Geophys. Res. Oceans, 118, 1508-1519, doi:10.1002/jgrc.20151.

Charney, J. (1955), "The Use of the Primitive Equations of Motion in Numerical Prediction", Tellus VII, 7(1), 22-26, https://doi.org/10.1111/j.2153-3490.1955.tb01138.x.

Chavanne, C. P., K. J. Heywood, K. W. Nicholls, and I. Fer (2010), “Observations of the Antarctic Slope undercurrent in the southeastern Weddell Sea", Geophys. Res. Lett., 37(L13601), doi:10.1029/2010GL043603.

Chelton, D. B., M. G. Schlax, R. M. Samelson, and R. A. de Szoeke (2007), "Global observations of large oceanic eddies", Journal of Geophysical Research Letters, 34(L15606), doi:10.1029/2007GL030812.

Chelton, D. B., M. G. Schlax, and R. M. Samelson (2011), “Global observations of nonlinear mesoscale eddies", Progress in Oceanography, 91(2), 167-216, doi:10.1016/j.pocean.2011.01.002.

Dewar, W. K., and P. D. Killworth (1995), "On the stability of oceanic rings", Journal of Phys. Oceanogr, 25, 1467-1487. 
Doglioli, A. M., B. Blanke, S.Speich, and G. Lapeyre (2007), “Tracking coherent structures in a regional ocean model with wavelet analysis: Application to Cape Basin eddies", Journal of Geophysical Research: Oceans, 112(C5043), doi:10.1029/2006JC003952.

Douglass, E. M., and J. G. Richman (2015), “Analysis of ageostrophy in strong surface eddies in the Atlantic Ocean", Journal of Geophysical Research: Oceans, 120, 14901507, doi:10.1002/ 2014JC010350.

Endlich, R. (1961), "Computation and uses of gradient winds", Mon. Weather Rev., 89, 187-191, https:// doi.org/10.1006/rwos.2001.0376.

Escudier, R., B. Mourre, M. Juza, and J. Tinto (2016), "Subsurface circulation and mesoscale variability in the Algerian subbasin from altimeter-derived eddy trajectories", Journal of Geophysical Research: Oceans, 121(8), 6310-6322, doi:10.1002/2016JC011760.

Flexas, M., D. Gomis, S. Ruiz, A. Pascual, and P. Leon (2006), "In situ and satellite observations of the eastward migration of the Western Alboran Sea Gyre", Progress in Oceanography, 70, 486-509.

Garreau, P., F. Dumas, S. Louazel, A. Stegner, and B.Le Vu (2018), "High resolution in situ observations and tracking of a dual core anticyclonic eddy in the Algerian Basin", J. Geophys. Res. Oceans., 123, 9320-9339, https://doi.org/10.1029/2017JC013667.

Gomis, D., S. Ruiz, and M. Pedder (2001), "Diagnostic analysis of the 3D ageostrophic circulation from a multivariate spatial interpolation of CTD and ADCP data.", Deep-Sea Research I, 48, 269-295.

Hamad, N., C. Millot, and I. Taupier-Letage (2006), "The surface circulation in the Eastern basin of the Mediterranean Sea", SCIENTIA MARINA, 70(3), 457-503.

Hasegawa, D., H. Yamazaki, R. G. Lueck, and L. Seuront (2004), "How islands stir and fertilize the upper ocean", Geophys. Res. Lett., 31(L16303), doi:10.1029/2004GL020143.

Horton, C., J. Kerling, G. Athey, J. Schmitz, and M.Clifford (1994), “Airborne expendable bathythermograph surveys of the eastern Mediterranean", Journal of Geophysical Research: Oceans, 99(C5), 9891-9905, doi:10.1029/94JC00058.

Ioannou, A., A. Stegner, B. Le Vu, I. Taupier-Letage, and S. Speich (2017), "Dynamical Evolution of Intense Ierapetra Eddies on a 22 Year Long Period", Journal of Geophysical Research:Oceans, 122, https://doi.org/10.1002/2017JC013158. 
Isern-Fontanet, J., E. García-Ladona, and J. Font (2006), "Vortices of the Mediterranean Sea: An Altimetric Perspective", Journal of Physical Oceonography, 36(1), 87-103, doi: 10.1175/JPO2826.1,.

Iversen, T., and T. E. Nordeng (1982), “A Convergent Method for Solving the Balance Equation", Monthly Weather Review, 110, 1347-1353, https://doi.org/10.1175/15200493(1982)110<1347:ACMFST>2.0.CO;2.

Iversen, T., and T. E. Nordeng (1984), "Static initialization of primitive equation models on a bounded, extratropical region ", Tellus, 36(A), 21-29, https://doi.org/10.1111/j.1600-0870.1984.tb00219.x.

Klein, P., B. L. Hua, G. Lapeyre, X. Capet, S. L. Gentil, , and H. Sasaki (2008), "Upper ocean turbulence from high-resolution 3D simulations.", Journal of Phys. Oceanogr., 38, 1748-1763, doi:10.1175/ 2007JPO3773.1.

Knox, J. A., and P. R. Ohmann (2006), "Iterative solutions of the gradient wind equation", Comput. Geosci., 32, 656-662.

Lahaye, N., and V. Zeitlin (2015), "Centrifugal, barotropic and baroclinic instabilities of isolated ageostrophic anticyclones in the two-layer rotating shallow water model and their nonlinear saturation", J. Fluid Mech, 762, 5-34.

Laxenaire, R., S. Speich, B. Blanke, A. Chaigneau, C. Pegliasco, and A. Stegner (2018), "Anticyclonic eddies connecting the western boundaries of indian and atlantic oceans", J. Geophys. Res. Oceans, https://doi.org/10.1029/2018JC014270.

Lazar, A., A. Stegner, and E.Heifetz (2013), "Inertial instability of intense stratified anticyclones.part 1.Generalized stability criterion", Journal of Fluid Mechanics, 732, 457-484, doi:10.1017/jfm.2013.412.

LeTraon, P. Y., F. Nadal, and N. Ducet (1998), “An Improved Mapping Method of Multisatellite Altimeter Data", JOURNAL OF ATMOSPHERIC AND OCEANIC TECHNOL$O G Y, 15,522-533$.

Le Vu, B., A. Stegner, and T. Arsouze (2018), "Angular momentum eddy detection and tracking algorithm (ameda) and its application to coastal eddy formation", J. Atmos. Oceanic Technol, 35, 739-762, doi:10.1175/JTECH-D-17-0010.1.

Mahdinia, M., P. Hassanzadeh, P. S. Marcus, and C.-H. Jiang (2017), “Stability of 3d gaussian vortices in an unbounded, rotating, vertically-stratified,boussinesq flow: Linear analysis", Journal of Fluid Mech, 824, 97-134, doi:10.1017/jfm.2017.303. 
Mason, E., A. Pascual, and J. C. McWilliams (2014), “A New Sea Surface Height Based Code for Oceanic Mesoscale Eddy Tracking", Journal of Atmospheric and Oceanic Technology, 31, 1181-1188, doi:10.1175/JTECH-D-14-00019.1.

Matteoda, A., and S. Glenn (1996), “Observations of recurrent mesoscale eddies in the eastern Mediterranean", Journal of Geophysical Research, 101(C9), 20,687-20,709, doi:10.1029/96JC01111.

McIntyre, M. (2015), "Balanced Flow", Encyclopedia of Atmospheric Sciences, 2nd edition, 2, 298-303.

Menna, M., P. Poulain, G. Zodiatis, and I. Gertman (2012), "On the surface circulation of the Levantine sub-basin derived from Lagrangian drifters and satellite altimetry data", Deep-Sea Research, 65, 46-58, doi:10.1016/j.dsr.2012.02.008.

Mkhinini, N., A. L. S. Coimbra, A. Stegner, T. Arsouze, I. Taupier-Letage, and K. Beranger (2014), "Long-lived mesoscale eddies in the eastern Mediterranean Sea: Analysis of 20 years of AVISO geostrophic velocities", Journal of Geophysical Research: Oceans, 119(12), 8603-8626, dOI:10.1002/2014JC010176.

Nencioli, F., C. Dong, T. Dickey, L. Washburn, and J. McWilliams (2010), “A vector geometry-based eddy detection algorithm and its application to a highresolution numerical model product and high-frequency radar surface velocities in the southern california bight", Journal of Atmos. Oceanic Technol., 27(3), 564-579, doi:10.1175/2009JTECHO725.1.

Paduan, J., and L. Washburn (2013), "High-frequency radar observations of ocean surface currents", J. Mar.Sci., doi: 10.1146/annurev-marine-121211-172315.

Penven, P., I. Halo, S. Pous, and L. Marié (2014), "Cyclogeostrophic balance in the Mozambique Channel", Journal of Geophysical Research: Oceans, 119(2), 1-14, doi:10.1002/2013jc009528.

Pessini, F., A. Olita, Y. Cotroneo, and A. Perilli (2018), "Mesoscale Eddies in the Algerian Basin: do they differ as a function of their formation site?", Ocean Sci. Discuss., https://doi.org/10.5194/os-2017-93.

Pessini, F. a. (2019), "Life history of an anticyclonic eddy in the Algerian basin from altimetry data, tracking algorithm and in situ observations", Journal of Marine Systems.

Puillat, I., I. Taupier-Letage, and C. Millot (2002), “Algerian Eddies lifetime can near 3 years", Journal of Marine Systems, 31(4), 245-259, doi:10.1016/S0924-7963(01) 00056-2. 
Qiu, B., S. Chen, P. Klein, H. Sasaki, and Y. Sasai (2014), "Seasonal mesoscale and submesoscale eddy variability along the North Pacific Subtropical Countercurrent", Journal of Phys. Oceanogr, 12, 3070-3098, doi:10.1175/JPO-D-14-0071.1.

Robinson, A. R., W. G. Leslie, A. Theocharis, and A. Lascaratos (2001), "Mediterranean Sea circulation", Encyclopedia of Ocean Sciences,J. H. Steele, Ed., Academic Press, pp. 1689-1705, https:// doi.org/10.1006/rwos.2001.0376.

Roullet, G., and K. P. (2010), "Cyclone-anticyclone asymmetry in geophysical turbulence", Phys. Rev. Lett., 104(218501), doi:10.1103/PhysRevLett.104.218501.

Schaeffer, A., A. Gramoulle, M. Roughan, and A. Mantovanelli (2017), "Characterizing frontal eddies along the East Australian Current from HF radar observations", J. Geophys. Res. Oceans, https://doi.org/10.1002/2016JC012171.

Souza, J. M. A., C. de Boyer Montégut, and P.-Y. L. Traon (2011), "Comparison between three impleamentations of automatic identification algorithms for the quantification and characterization of mesoscale eddies in the South Atlantic Ocean", Ocean Sci., 7, 317334, https://doi.org/10.5194/os-7-317-2011.

Stegner, A., and D. Dritschel (2000), "A numerical investigation of the stability of isolated shallow water vortices", Journal of Physical Oceanography, 30(10), 2562-2573, doi:10.1175/1520-0485(2000)030<2562:ANIOTS>2.0.CO;2.

Sutyrin, G., A. Stegner, I. Taupier-Letage, and S. Teinturier (2009), "Amplification of a Surface-Intensified Eddy Drift along a Steep Shelf in the Eastern Mediterranean Sea", Journal of Physical Oceonography, 39, 1729-1741, doi:10.1175/2009JPO4106.1.

Uchida, H., S. Imawaki, and J. Hu (1998), “Comparison of Kuroshio surface velocities derived from satellite altimeter and drifting buoy data", J. Oceanogr., 54, 115-122.

Viudez, A. (1997), "An Explanation for the Curvature of the Atlantic Jet past the Strait of Gibraltar", Journal of Physical Oceanography, 27, 1804-1810.

Viudez, A., J. Tintoré, and R. Haney (1996a), "Circulation in the Alboran Sea as determined by quasi-synoptic hydrographic observations. i: Three dimensional structure of the two anticyclonic gyres", Journal of Physical Oceanography, 26, 684-705.

Viudez, A., R. Haney, and J. Tintoré (1996b), "Circulation in the Alboran Sea as determined by quasi-synoptic hydrographic observations.ii: Mesoscale ageostrophic motion diagnosed through density dynamical assimilation", Journal of Physical Oceanography, $26,706-724$ 

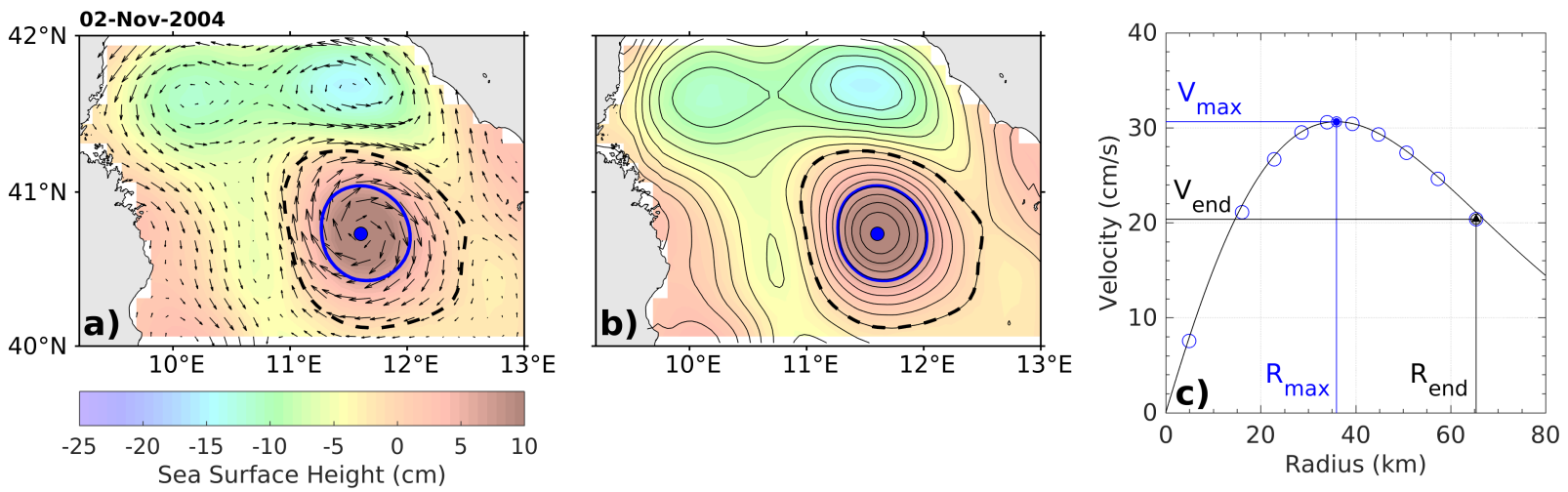

Figure 1. Principle of the automatic eddy detection algorithm AMEDA. The characteristic contour (solid blue line) and the last contour (black dashed line) are calculated from the surface velocity field (a) for an large anticyclone located at the east of Sardinia. The background colors correspond to the ADT map while the black vectors to the surface geostrophic velocities. The streamlines associated with the velocity field are plotted in (b) and also the correspondence with the characteristic contour (solid blue line) and the last closed contour (black dashed line). The mean velocity profile $\langle V\rangle=F(\langle R\rangle)$ deduced from the streamlines analysis and the characteristic eddy radii $R_{\max }$ and $R_{\text {end }}$ are plotted in (c).
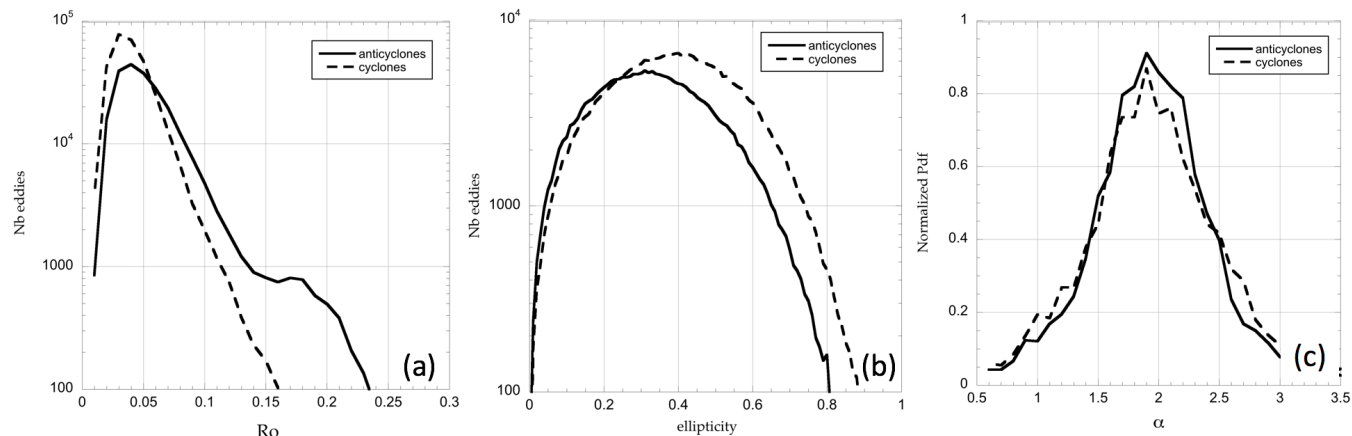

Figure 2. Probability distributions of the vortex Rossby number (a), the ellipticity $\varepsilon$ (b) and the steepness parameter $\alpha$ (c) for cyclones (solid line) and anticyclones (dashed line) in the Mediterranean sea for the 20002015 period. We consider here only geostrophic mesoscale eddies having a characteristic radius larger than $R_{\max } \geq 18 \mathrm{~km}$. The steepness parameter $\alpha$ was computed here only for quasi circular eddies $(\varepsilon<0.2)$. 

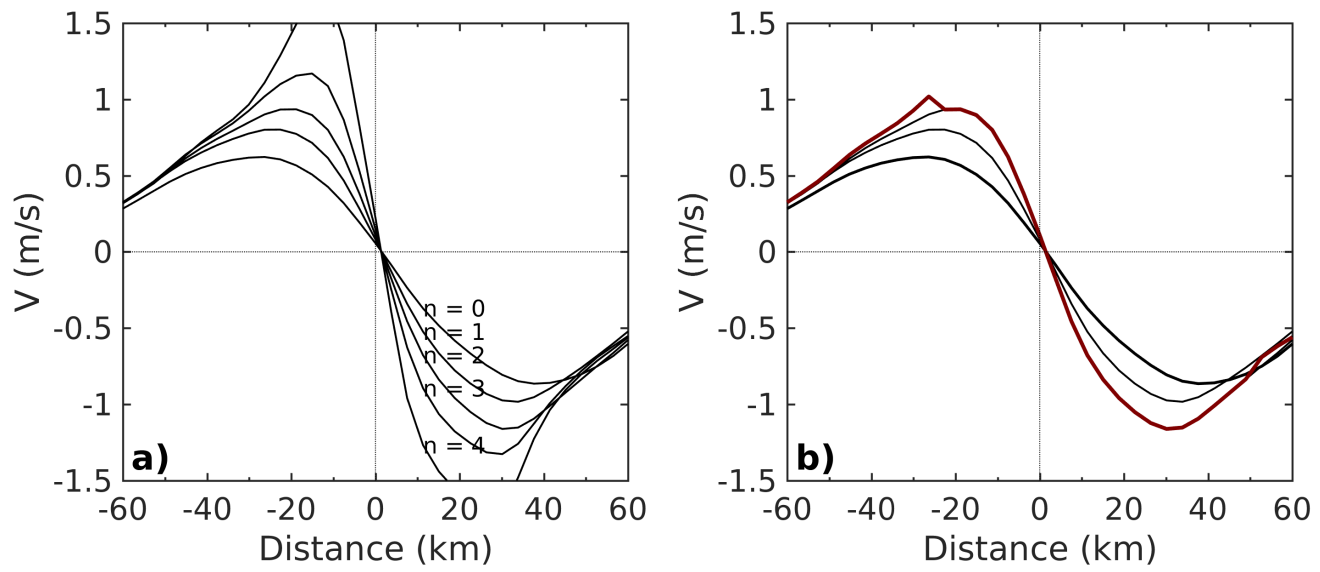

Figure 3. Modifications of the meridional velocity profile of the Alboran eddy (depicted Figure 4) along the longitude axis at each step of the iterative process Eqn. (6) for the free iteration (a) and for the constrained iteration (b). The vortex Rossby number, of the initial geostrophic eddy (bold profile in (b)), is 0.24 while at the end of the constrain iteration (red profile in (b)) it reaches $R o=0.48$.
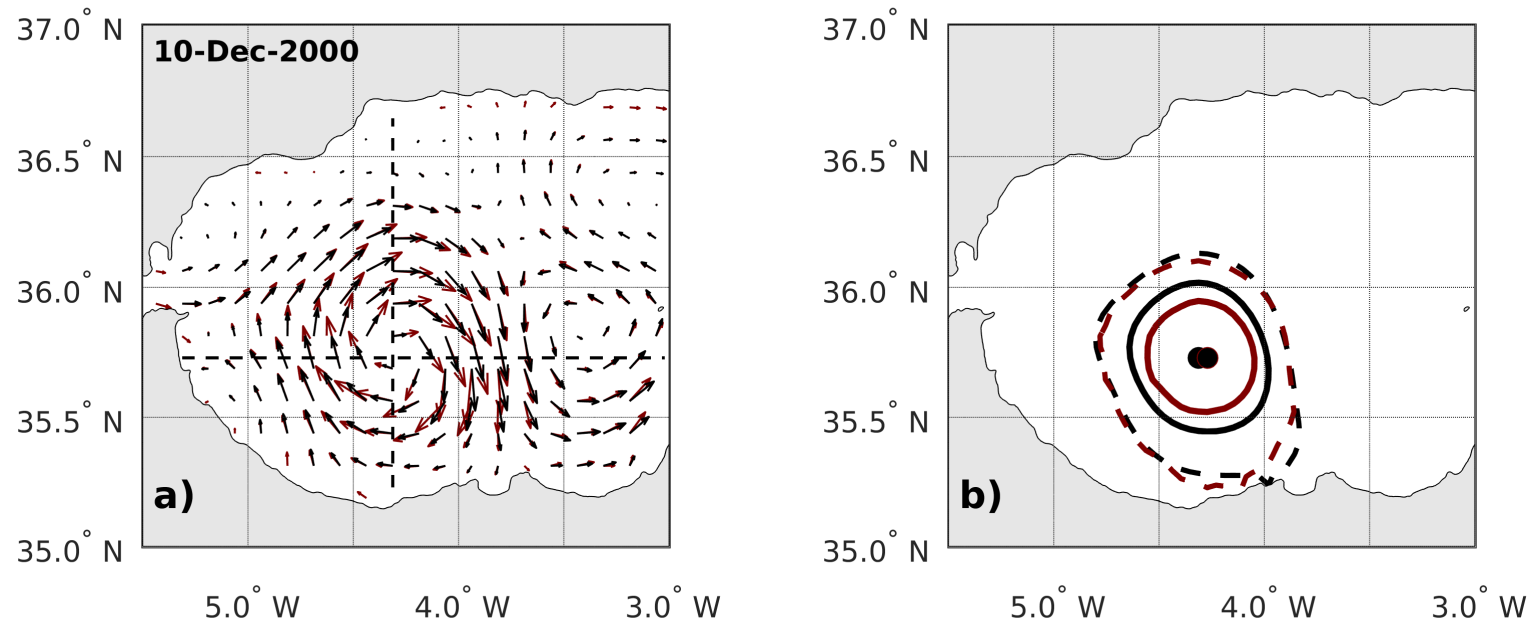

Figure 4. Example of the cyclogeostrophic corrections (red) applied to the AVISO/DUACS surface geostrophic velocity field (black) on the Alboran anticyclone in December 2000 (a). The characteristic eddy contour computed by the AMEDA algorithm (b) is also modified by the cyclogeostrophic correction (red) in comparison with the initial contour computed from the geostrophic field (black) as shown in Figure 3. 

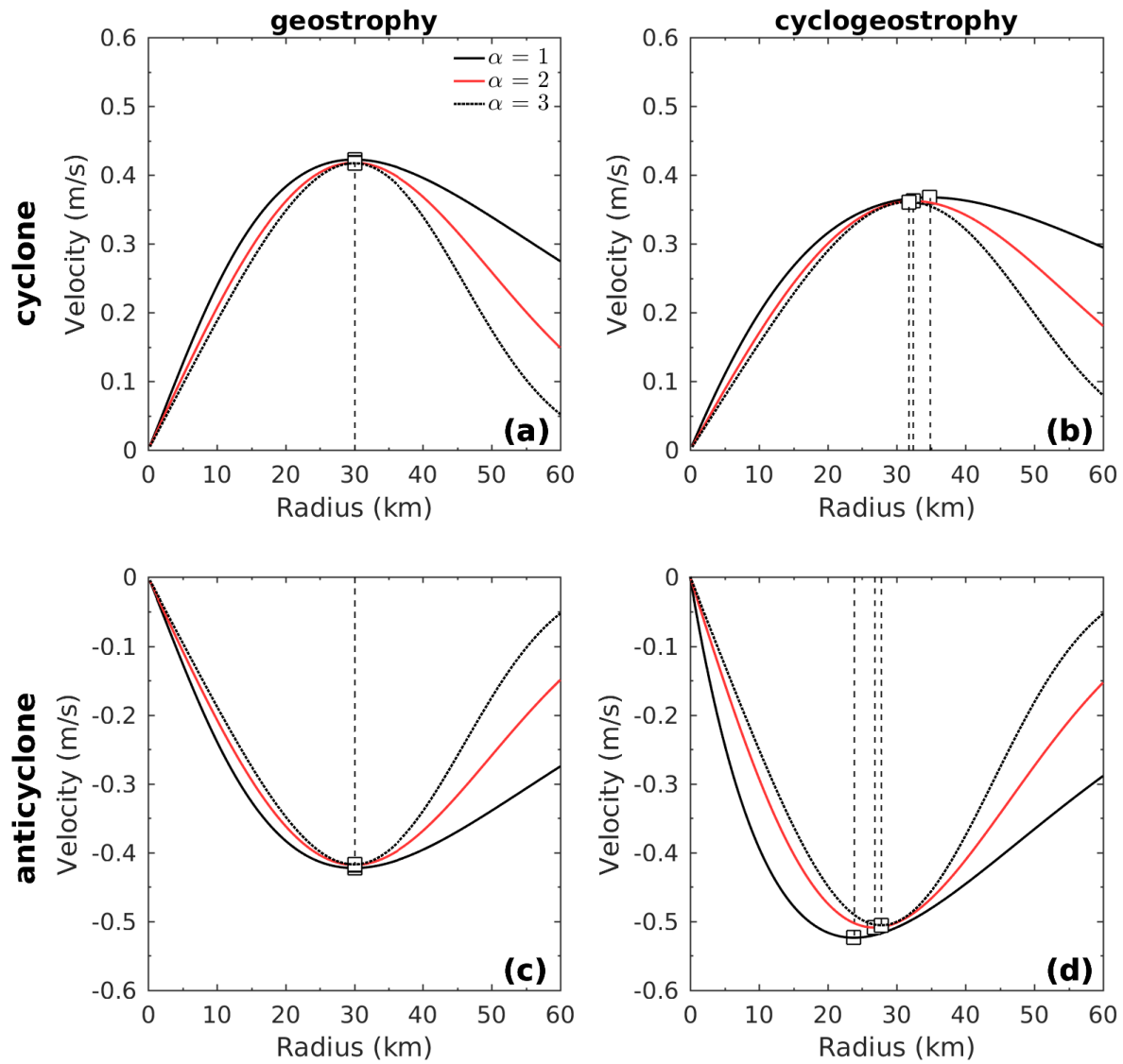

Figure 5. Comparison of geostrophic vs. cyclogeostrophic velocity profiles of both cyclonic and anticyclonic eddies having the same geostrophic Rossby number $\left(R o_{g}=0.14\right)$ but distinct steepness parameters: $\alpha=1$ (black solid line), a Gaussian eddy $\alpha=2$ (red line) and $\alpha=3$ (black dashed line). 

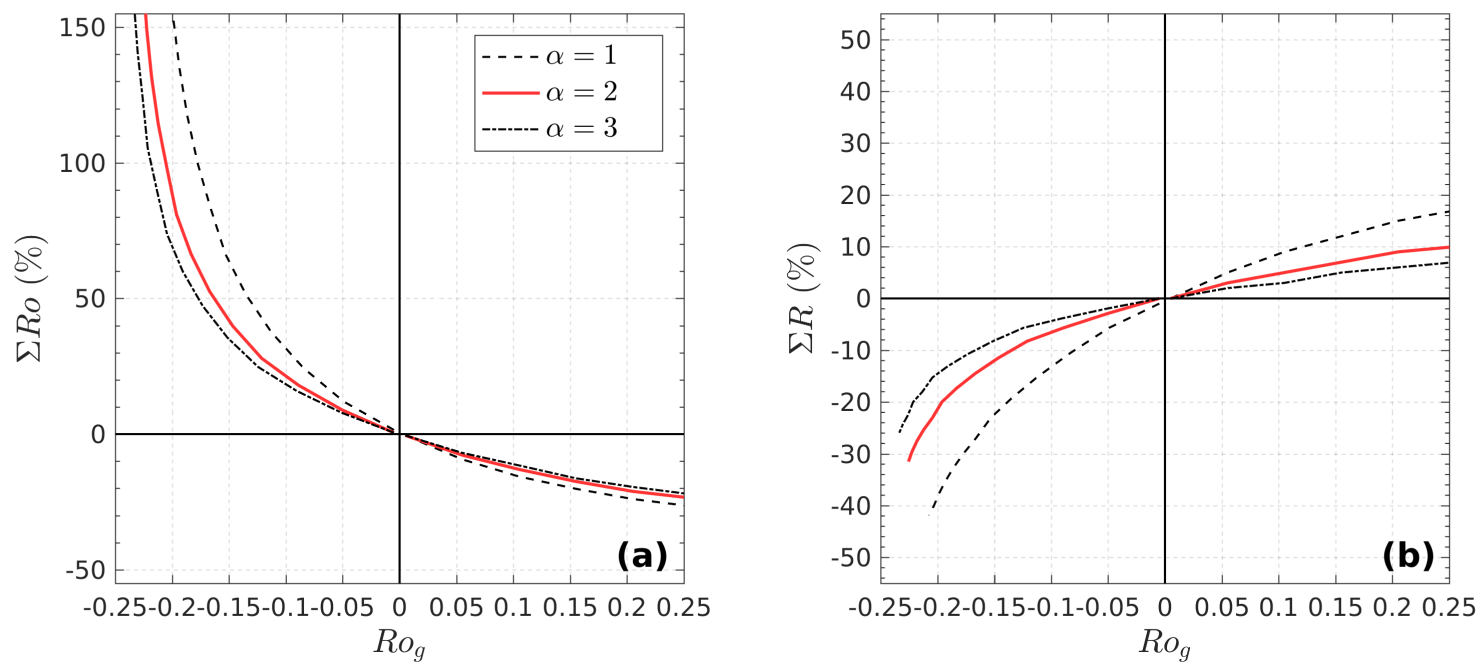

Figure 6. Percentages of the relative errors, between the cyclogeostrophic and the geostrophic vortex solution, are plotted for the vortex Rossby number (a) and the characteristic eddy radius $R_{\max }$ (b). Negative (positive) Rossby numbers correspond here to anticyclonic (cyclonic) eddies. Various circular eddies having distinct shape ( $\alpha=1$ dotted line, $\alpha=2$ solid line and 3 dashed line) and intensity $\left(-0.25<R o_{g} \leq 0.25\right)$ are considered.
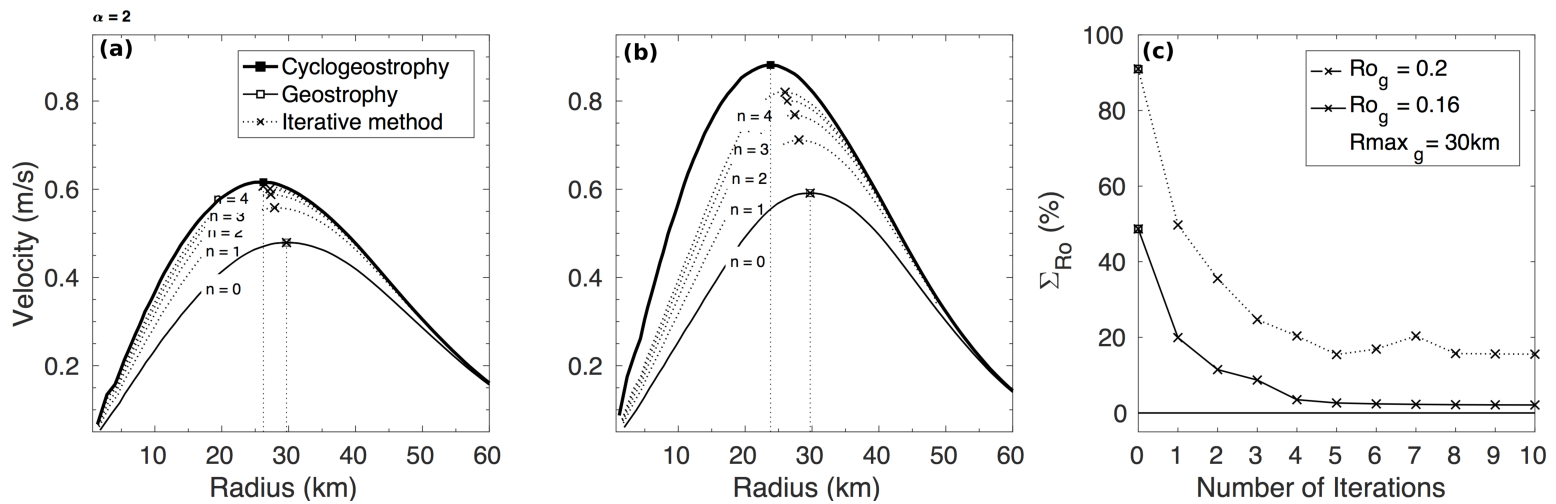

Figure 7. Accuracy of the iteration scheme Eqn. (6) applied to two geostrophic gaussian anticyclones $(\alpha=2)$ having the same radius, $R_{\max }=30 \mathrm{~km}$, but distinct Rossby number: $R o_{g}=0.16$ (a) and $R o_{g}=0.2$ (b). The initial geostrophic profiles $V_{g}(r)$ are plotted with thin dashed lines, while the targeted velocity profiles $V_{\theta}(r)$, solution of the cyclogeostrophic equation Eqn. (7), are plotted with a thick black line. (c) Relative error $\left(\Sigma_{R o}=\left(R o-R o_{i}\right) / R o_{i}\right)$ between the Rossby number reached at every iterative step $\left(R o_{i}\right)$ and the corresponding exact cyclogeostrophic solution $(R o)$ for the two gaussian anticyclones. 

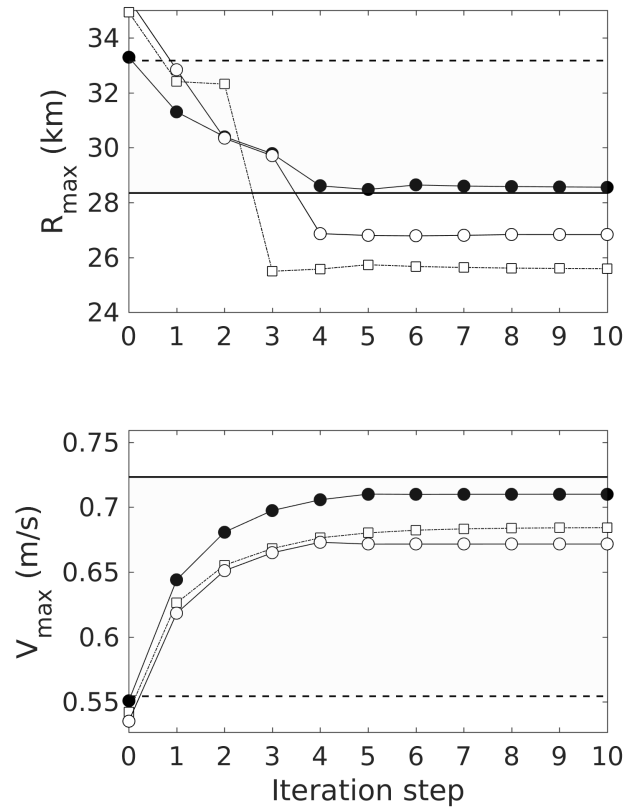

Figure 8. Impact of the initial interpolation on the evolution of the characteristic radius $R_{\max }$ and the speed radius $V_{\max }$ at each step of the iteration scheme Eqn. (6) for an initial geostrophic gaussian eddy ( $\alpha=2$ ). The horizontal dashed lines correspond to the initial geostrophic solution. The horizontal solid lines correspond to the targeted values of the cyclogeostrophic solution. Three cases are plotted: no initial interpolation (open circle), a linear interpolation at $1 / 24^{\circ}$ (open square) and a cubic interpolation (filled circle) at $1 / 24^{\circ}$. 

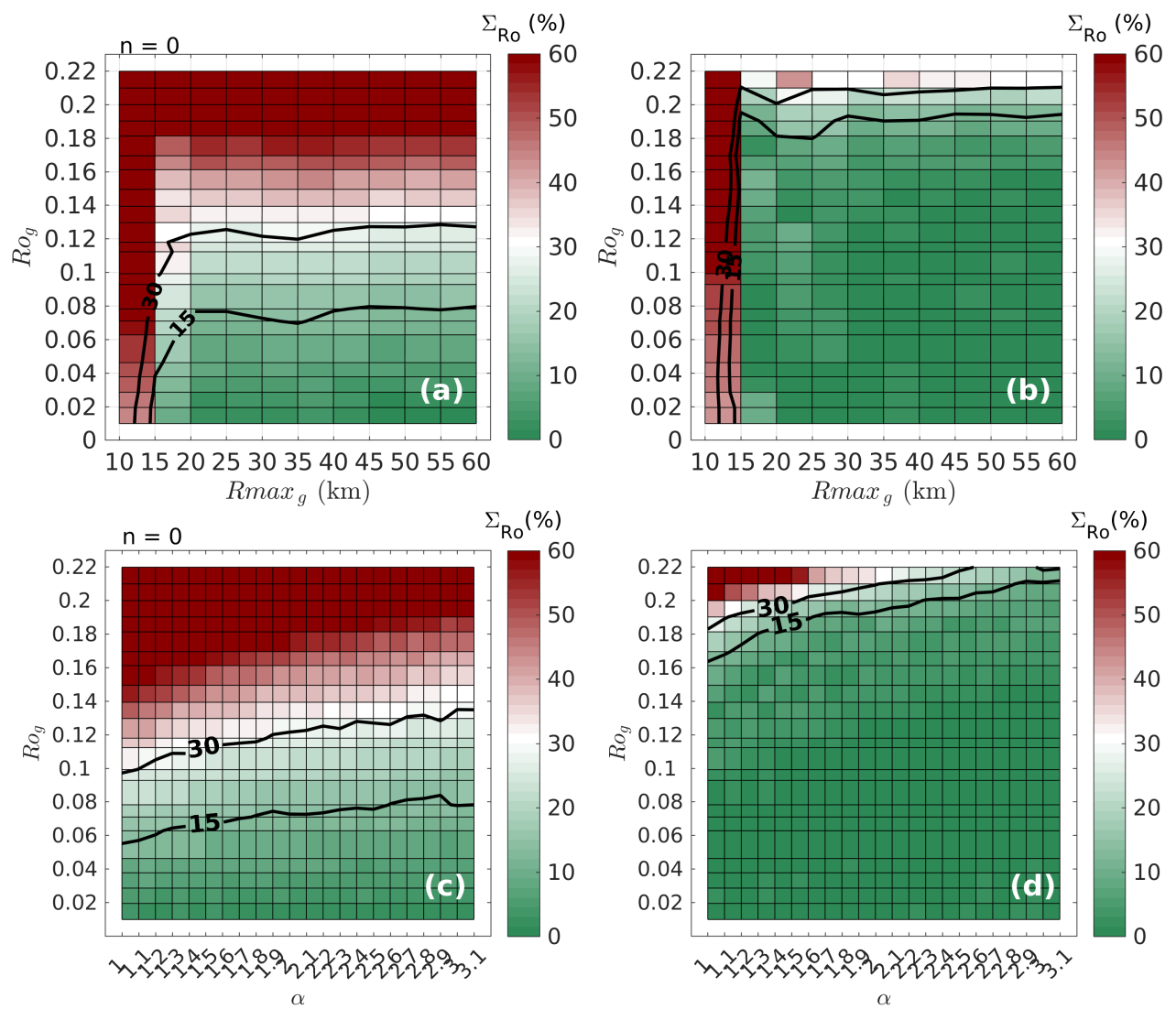

Figure 9. Initial relative error on the vortex Rossby number between geostrophic and cyclogeostrophic circular anticyclones of various intensity $\left(R o_{g}\right)$, sizes (a), or profiles (b) when there is no correction on the initial geostrophic velocity field. Relative error $\left(\Sigma_{R o}=\left(R o-R o_{i}\right) / R o_{i}\right)$ between the Rossby number reached at the end of the iterative scheme $\left(R o_{i}\right)$ and the one corresponding to the exact cyclogeostrophic solution $(R o)$ for the same range of initial parameters $(b, d)$. 


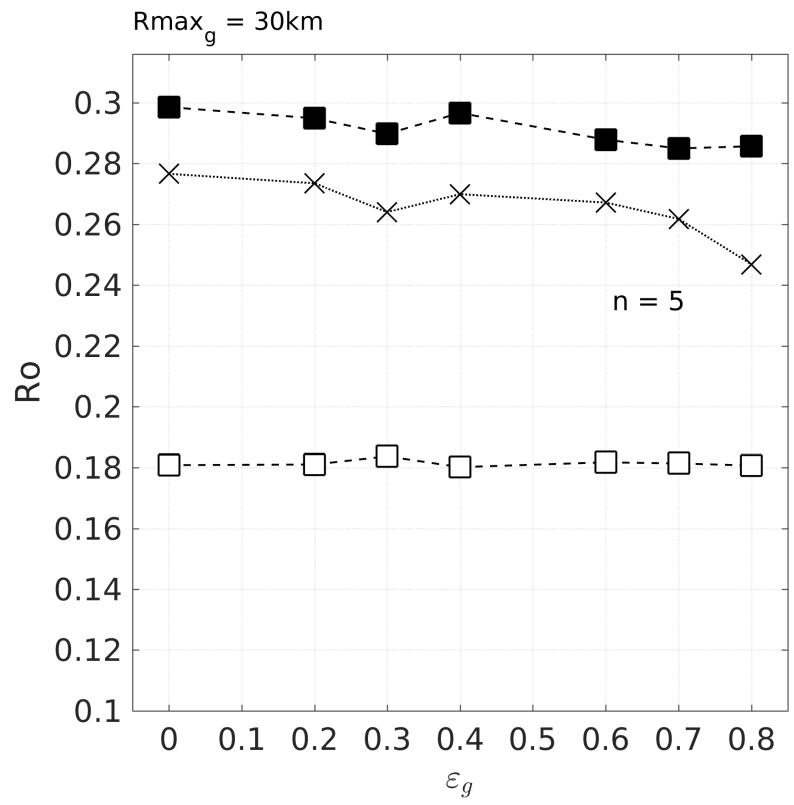

Figure 10. Evolution of the vortex Rossby numbers for geostrophic (open square) and cyclogeostrophic (filled square) elliptical anticyclones as a function of their ellipticity $\varepsilon=1-b / a$, where $a$ and $b$ are respectively the semi-major and semi-minor axis. The elliptical velocity fields were obtained from the deformation (Eqn. (10-13)) of a circular Gaussian velocity profile having a characteristic radius $R_{\max }=30 \mathrm{~km}$ and a maximum azimuthal speed $V_{\max }=0.9 \mathrm{~m} \mathrm{~s}^{-1}$. The vortex Rossby numbers of the elliptical eddies obtained by the iterative scheme Eqn. (6) applied to the gesotrophic solutions are plotted with crosses. 

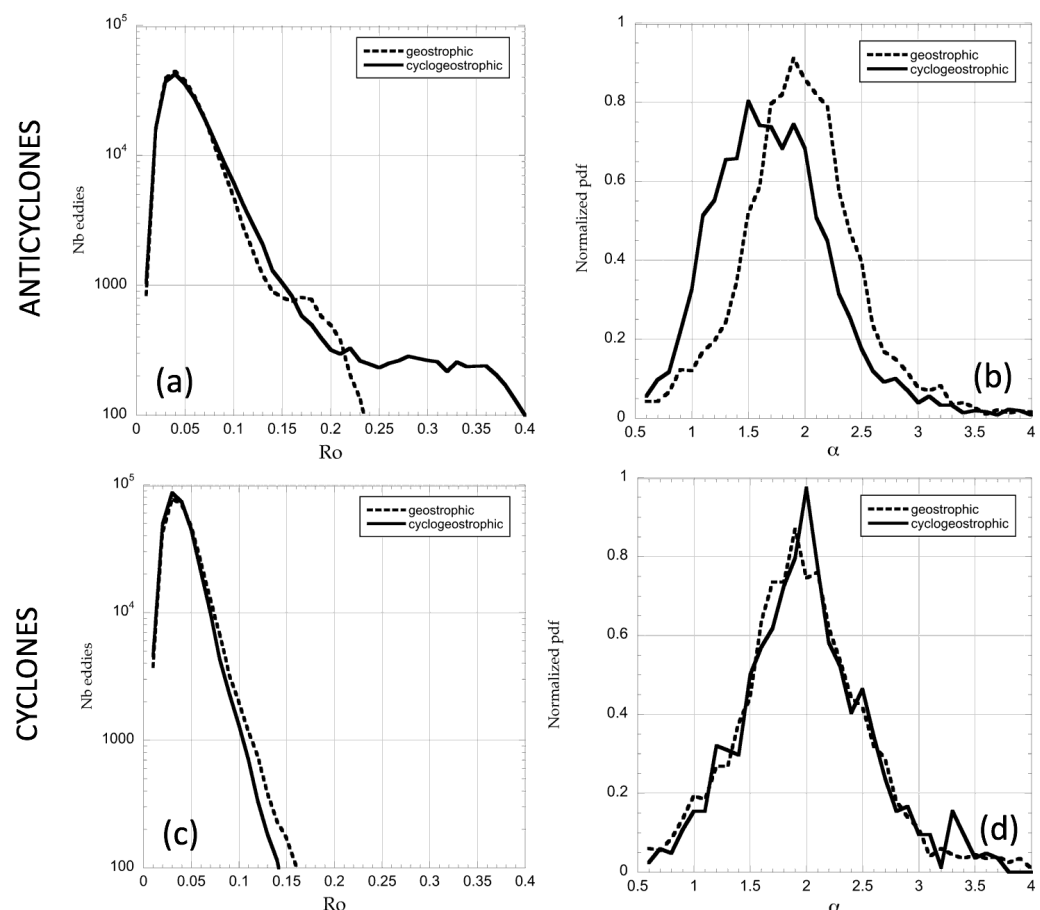

Figure 11. Probability distribution function of the vortex Rossby numbers $R o_{g}$ and $R o(\mathrm{a}, \mathrm{c})$ and the steepness parameters $\alpha(\mathrm{b}, \mathrm{d})$ of the mesoscale eddies detected by the AMEDA algorithm on the AVISO/DUACS geostrophic velocity field (dashed line) and on the corrected velocity field where cyclogeostrophic components are estimated (black solid line). 
$0^{\circ}$

$30^{\circ} \mathrm{E}$

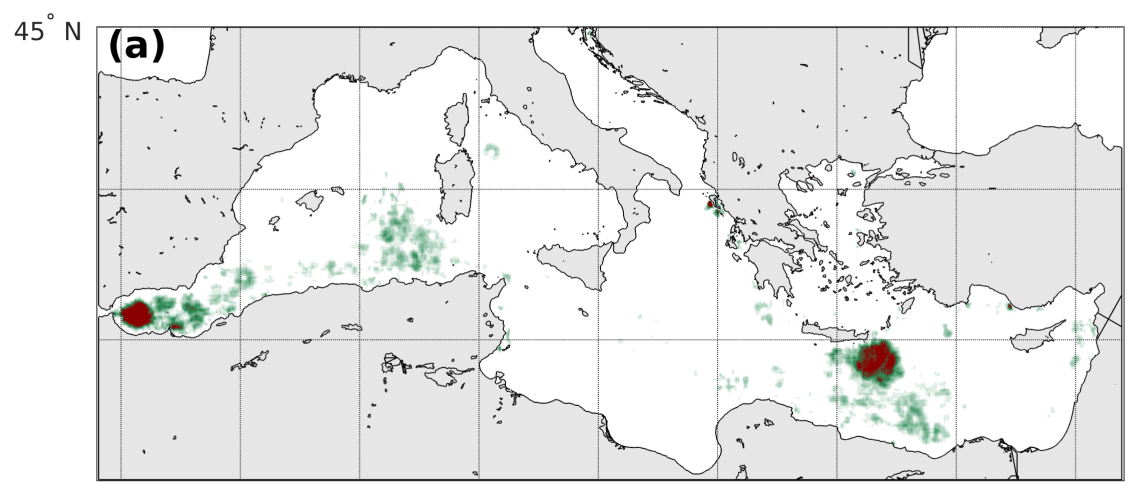
0.5

$0.4 \widehat{\tau}$ छे $0.3 \overline{\bar{\sigma}}$ $0.2 \stackrel{\text { ù }}{=}$
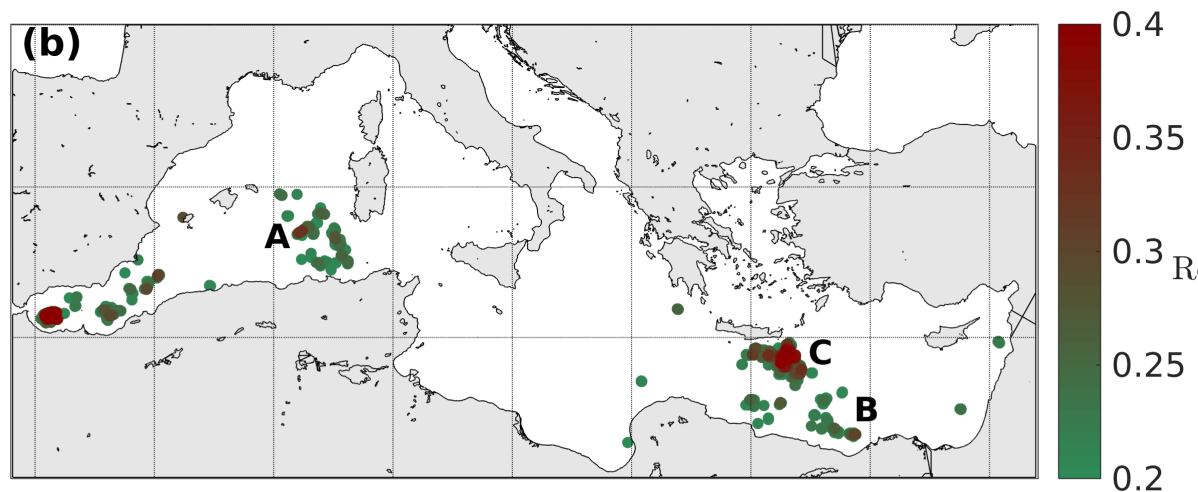

Figure 12. The localization of the maximal velocity correction, averaged for 5 days at each grid point, during the 15 year period (2000-2015) is plotted in the upper panel (a). Velocity corrections having an amplitude $\left\|\mathbf{V}-\mathbf{V}_{g}\right\|$ below $10 \mathrm{~cm} \mathrm{~s}^{-1}$ are not plotted. The location of eddies detected by the AMEDA algorithm (once the cyclostrophic correction is applied) having a vortex Rossby number higher than 0.2 are plotted in the lower panel (b). 

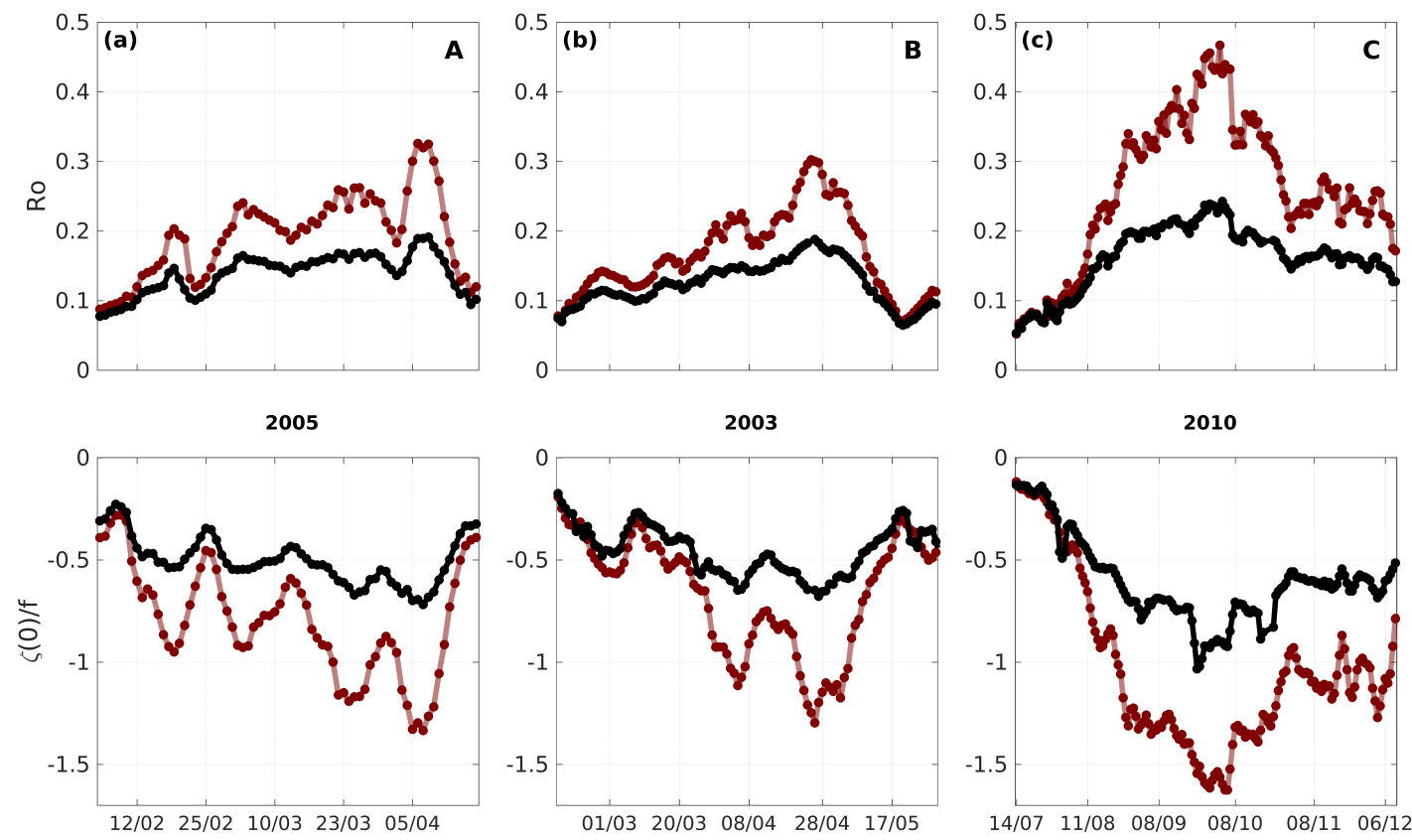

Figure 13. Temporal evolution of the vortex Rossby number Ro and the relative core vorticity $\zeta(0) / f$ for a) an Algerian Eddy (AE) detected in 2005 b) a Libyo-Egyptian eddy detected in 2003 and c) for an Ierapetra Eddy (IE) detected in 2010. The characteristics of the mesoscale eddies are illustrated with the black filled circles as detected by the AMEDA algorithm applied on the AVISO/DUACS geostrophic velocity fields and with the filled red circles when applied on the corrected cyclogeostrophic velocity fields. 

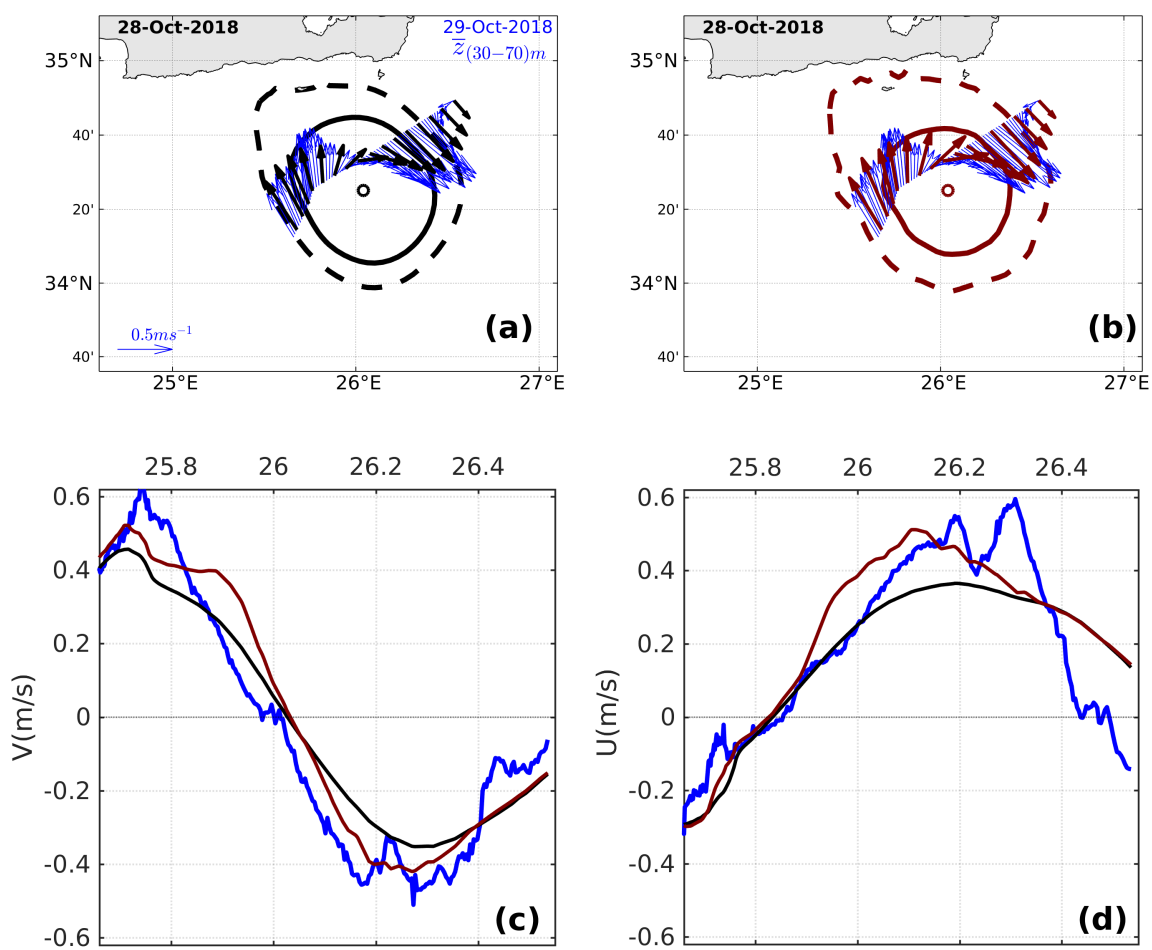

Figure 14. Comparison between the geostrophic surface velocities provided by the AVISO/DUACS product (black lines or arrows) and the VMADCP in-situ measurements (blue lines or arrows) performed the October 29, 2018. The cyclogeostrophic velocity field obtained by the iterative method Eqn. (6) is plotted in red. The upper panels show the surface geostrophic (a) or the cyclogeostrophic (b) velocity vectors along the boat trajectory in comparison with the VMADCP measurements. The characteristic contours (solid lines) and the last closed streamlines (dashed lines) computed by the AMEDA algorithm are both plotted for the geostrophic (in black, panel (a)) and the cyclogeostrophic (in red, panel (b)) surface velocity fields. The meridional and the latitudinal velocity profiles, of the geostrophic (black), the cyclogeostrophic (red) and the in-situ measurements (blue), are plotted respectively in the lower panels (c) and (d). 

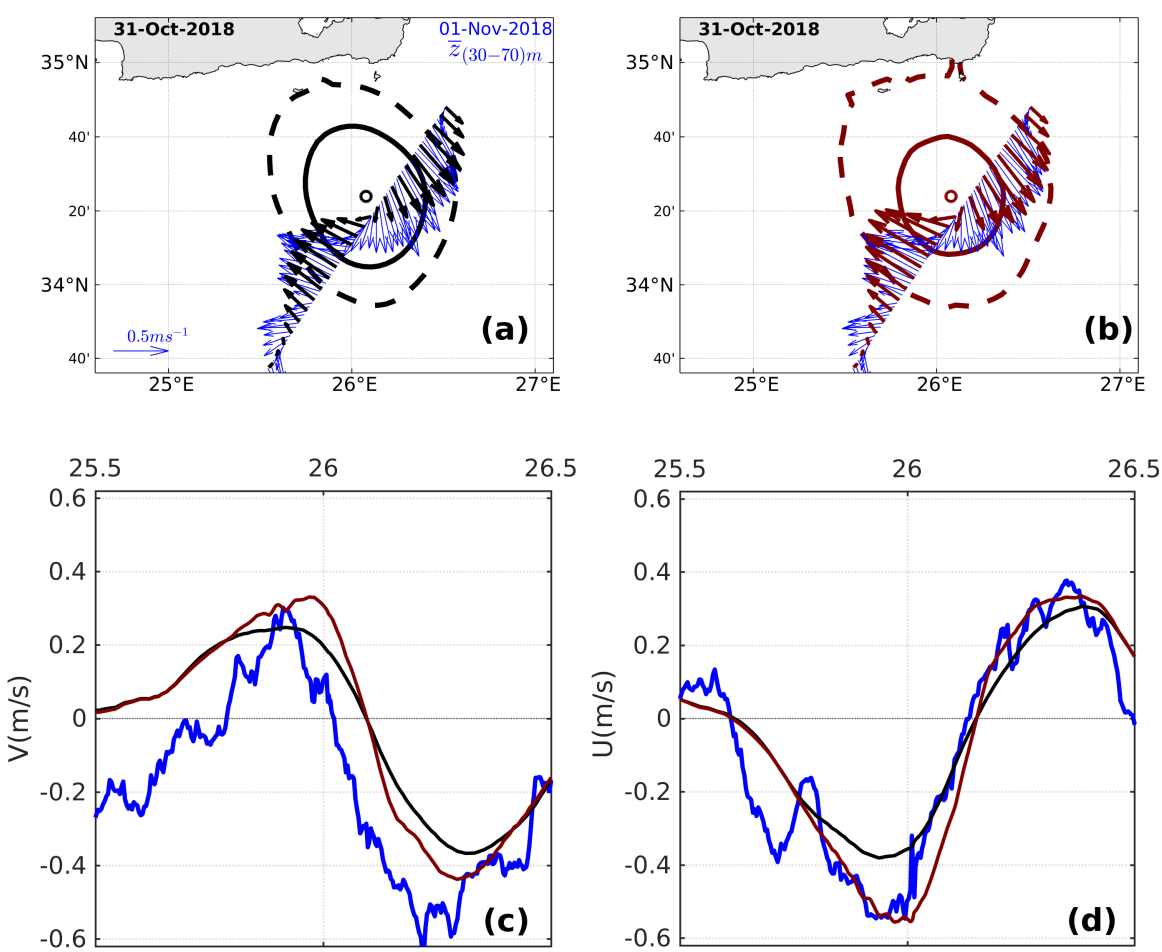

Figure 15. Comparison between the geostrophic surface velocity provided by the AVISO/DUACS product (black lines or arrows) and the VMADCP in-situ measurements (blue lines or arrows) performed on November 1, 2018. The panels are in the form identical to Figure 14. 

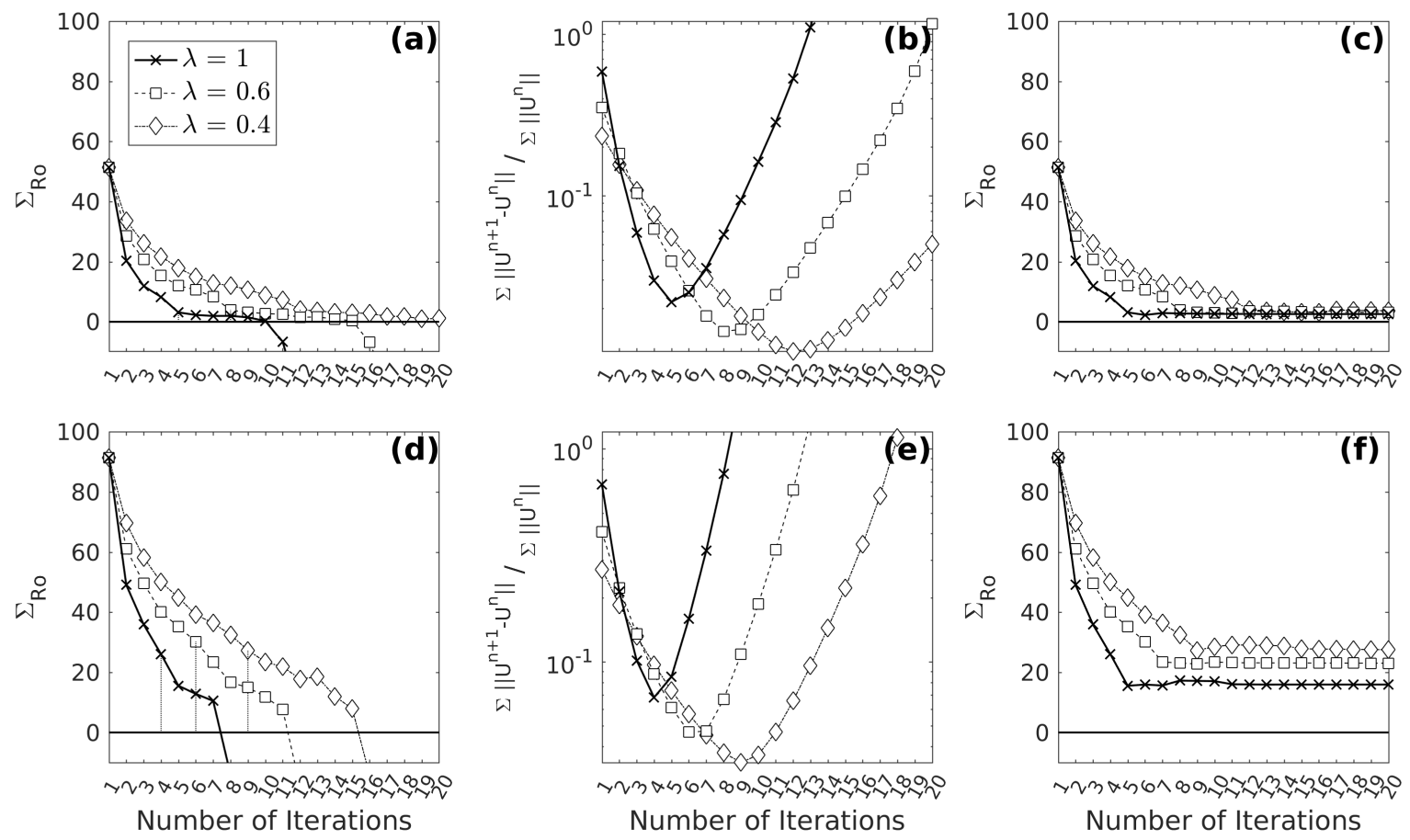

Figure A.1. Accuracy of the different iterative schemes (Eqn.(A.1) - Eqn(A.2)) applied on two geostrophic anticyclones with $R o_{g}=0.16$ in the upper panels and $R o_{g}=0.2$ in the lower panels as described in Figure 7. The classical iterative method (crosses) and the iterative method with the under-relaxation parameter $\lambda=0.4$ (diamonds) and $\lambda=0.6$ (squares) are illustrated with the different markers. The Relative error $\left(\Sigma_{R o}=\left(R o-R o_{i}\right) / R o_{i}\right)$ between the Rossby number reached at every iteration step $\left(R o_{i}\right)$ and the corresponding exact cyclogeostrophic solution $(R o)$ is illustrated in panels a) and b). The normalized residual drop of the velocity norm is shown in panels b) and e) at every iteration step. The relative error $\Sigma_{R o}$ of the constrained iterative schemes is shown in panels c) and f). 